\title{
Observation of Electroweak Production of Same-Sign $W$ Boson Pairs in the Two Jet and Two Same-Sign Lepton Final State in Proton-Proton Collisions at $\sqrt{s}=13 \mathrm{TeV}$
}

\author{
A. M. Sirunyan et al. \\ (CMS Collaboration)
}

(Received 18 September 2017; published 22 February 2018)

\begin{abstract}
The first observation of electroweak production of same-sign $W$ boson pairs in proton-proton collisions is reported. The data sample corresponds to an integrated luminosity of $35.9 \mathrm{fb}^{-1}$ collected at a center-ofmass energy of $13 \mathrm{TeV}$ with the CMS detector at the LHC. Events are selected by requiring exactly two leptons (electrons or muons) of the same charge, moderate missing transverse momentum, and two jets with a large rapidity separation and a large dijet mass. The observed significance of the signal is 5.5 standard deviations, where a significance of 5.7 standard deviations is expected based on the standard model. The ratio of measured event yields to that expected from the standard model at leading order is $0.90 \pm 0.22$. A cross section measurement in a fiducial region is reported. Bounds are given on the structure of quartic vector boson interactions in the framework of dimension- 8 effective field theory operators and on the production of doubly charged Higgs bosons.
\end{abstract}

DOI: 10.1103/PhysRevLett.120.081801

The standard model (SM) of particle physics provides an accurate description of observations from many accelerator- and nonaccelerator-based experiments. The discovery of a Higgs boson [1-3] confirmed that $W$ and $Z$ gauge bosons acquire mass using the Higgs mechanism. This discovery motivates further study of the mechanism of electroweak (EW) symmetry breaking through measurements of vector boson scattering (VBS) processes. Physics models beyond the SM predict enhancements in VBS through modifications of the Higgs sector or the presence of additional resonances [4,5].

The main goal of this analysis is to identify same-sign $W$ boson pairs produced in association with two jets purely via the electroweak interaction. Candidate events contain exactly two identified leptons (electrons or muons) of the same charge, moderate missing transverse momentum $\left(p_{\mathrm{T}}^{\mathrm{miss}}\right)$, and two jets with a large rapidity separation and a large dijet mass. The selection of same-sign lepton events reduces the contribution from the strong production of $W$ boson pairs, making the experimental signature an ideal topology for VBS studies.

Figure 1 shows representative Feynman diagrams for EW and quantum chromodynamics (QCD)-induced samesign $W$ boson pair production.

\footnotetext{
*Full author list given at the end of the article.

Published by the American Physical Society under the terms of the Creative Commons Attribution 4.0 International license. Further distribution of this work must maintain attribution to the author(s) and the published article's title, journal citation, and DOI. Funded by SCOAP .
}

An excess of events with respect to SM expectation could signal the presence of anomalous quartic gauge couplings (AQGCs) [6] or the existence of a new resonance, such as a doubly charged Higgs boson. Doubly charged Higgs bosons are predicted in Higgs sectors beyond the SM where weak isotriplet scalars are included $[7,8]$. They can be produced via vector boson fusion (VBF) and decay to pairs of same-sign $W$ bosons [9].

First experimental results for EW same-sign $W$ boson pair searches were reported by the ATLAS and CMS Collaborations based on data collected at $\sqrt{s}=8 \mathrm{TeV}$,
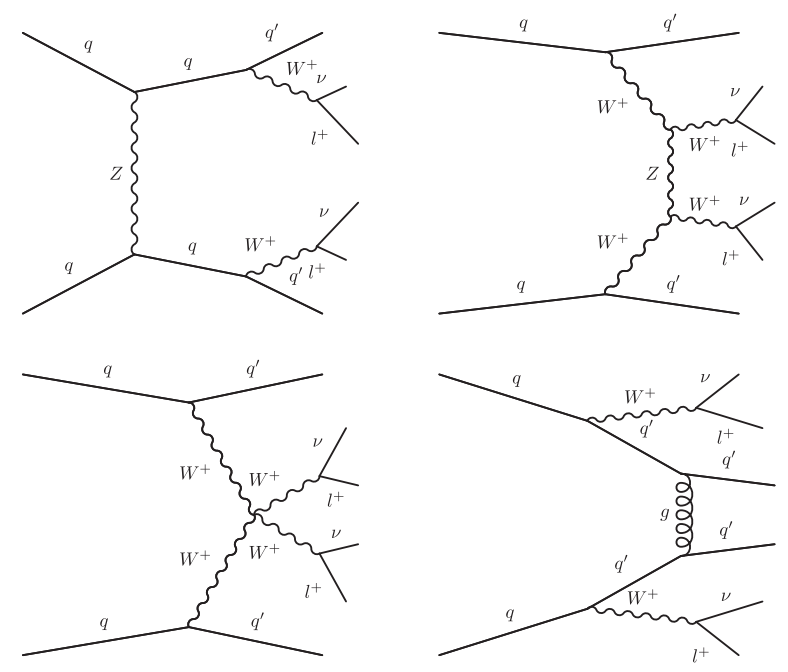

FIG. 1. Representative Feynman diagrams for single, triple, and quartic gauge couplings of the $\mathrm{EW}$-induced same-sign $W$ boson pair production (left, middle-left, middle-right) and QCD-induced background (right). 
corresponding to an integrated luminosity of approximately $20 \mathrm{fb}^{-1}[10,11]$. The observed significance is 3.6 (2.0) standard deviations for the ATLAS (CMS) study, where a significance of 2.8 (3.1) standard deviations is expected based on the SM prediction. This Letter presents a study of VBS in the same-sign $W$ boson pair final state at $\sqrt{s}=13 \mathrm{TeV}$. The data sample corresponds to an integrated luminosity of $35.9 \pm 0.9 \mathrm{fb}^{-1}$ collected with the CMS detector [12] at the CERN LHC in 2016.

The central feature of the CMS apparatus is a superconducting solenoid of $6 \mathrm{~m}$ internal diameter, providing a magnetic field of $3.8 \mathrm{~T}$. Within the solenoid volume are a silicon pixel and strip tracker, a lead tungstate crystal electromagnetic calorimeter, and a brass and scintillator hadron calorimeter, each composed of a barrel and two end cap sections. Forward calorimeters extend the pseudorapidity $\eta$ coverage provided by the barrel and end cap detectors. Muons are detected in gas-ionization detectors embedded in the steel flux-return yoke outside the solenoid. A more detailed description of the CMS detector, together with a definition of the coordinate system used and the relevant kinematic variables, can be found in Ref. [12].

The signal and background processes are simulated using the Monte Carlo (MC) generator MADGRAPH5_aMC@NLO 2.3.3 [13]. The same-sign $W$ boson pair samples are produced at leading order (LO) via diagrams with two or fewer QCD and up to six EW vertices. This includes two categories of diagrams: those with exactly two QCD vertices, which we refer to as QCD production, and those with no QCD vertices, which we refer to as EW production. We consider only the EW production as the signal in the analysis, whereas the QCD production is considered as background. This background is small and can be kinematically separated from the signal. The interference between the EW and QCD processes is at the level of a few percent in the signal region and is treated as a systematic uncertainty. The EW category includes diagrams with $W W W W$ quartic interactions and diagrams where two same-sign $W$ bosons scatter through the exchange of a Higgs boson, a $Z$ boson, or a photon. The $W Z$ and $Z Z$ production processes via $q \bar{q}$ annihilation and the $W \gamma$ process are generated at LO. The Drell-Yan, $Z \gamma, t \bar{t}, t \bar{t} W, t \bar{t} Z$, $W Z Z, W W Z, W W W$, and $Z Z Z$ are generated at next-toleading order (NLO). The simulated samples of background processes are normalized to the best theoretical prediction. The PYTHIA 8.205 [14] package is used for parton showering, hadronization, and the underlying event simulation, with tune CUETP8M1 [15,16]. The NNPDF 3.0 [17] set is used as the default set of parton distribution functions. The detector response is simulated by the GEANT4 package [18], based on a detailed description of the CMS detector. Proton-proton interactions occurring in the same beam crossing bin as the event of interest (pileup) are included in the simulation samples. The simulated pileup has a mean of approximately 27 and corresponds to the conditions observed in the $13 \mathrm{TeV}$ data collected in 2016.
The final states considered are $e^{+} e^{+} \nu_{e} \nu_{e} j j, e^{+} \mu^{+} \nu_{e} \nu_{\mu} j j$, $\mu^{+} \mu^{+} \nu_{\mu} \nu_{\mu} j j$, and their charge conjugates. The electrons and muons can be directly produced from a $W$ boson decay or from a $W$ boson with an intermediate $\tau$ lepton decay. A suite of single- and double-lepton triggers is used for this analysis [19]. The trigger efficiency for the signal process is larger than $99.8 \%$ after all other selection requirements are applied.

A particle-flow algorithm [20] is used to reconstruct observable particles in the event. It combines all subdetector information to reconstruct individual particles and identify them as charged and neutral hadrons, photons, and leptons. Electrons and muons are reconstructed by associating a track reconstructed in the silicon detectors either with a cluster of energy in the electromagnetic calorimeter [21] or a track in the muon system. The $p_{\mathrm{T}}^{\text {miss }}$ is defined as the magnitude of the negative vector sum of the transverse momenta of all reconstructed particles (charged and neutral) in the event modified by corrections to the energy scale of reconstructed jets.

The event selection aims to identify same-sign lepton events with the VBS topology, while reducing the contribution from QCD-induced same-sign $W$ boson pairs, the top quark, Drell-Yan, and $W Z$ background contributions. Two same-sign leptons, electrons or muons, with transverse momentum $p_{\mathrm{T}}>25(20) \mathrm{GeV}$ for the leading (trailing) lepton and $|\eta|<2.5$ (2.4) for electrons (muons) are required. Electrons and muons are required to be isolated from other charged and neutral particles in the event. Jets are reconstructed using the anti- $k_{T}$ clustering algorithm [22] with a distance parameter $R=0.4$, as implemented in the FASTJET package $[23,24]$. Events are required to contain at least two jets with $p_{\mathrm{T}}>30 \mathrm{GeV}$ and $|\eta|<5$.0. The VBS topology is targeted by requiring that the two highest $p_{\mathrm{T}}$ jets have a large dijet mass, $m_{j j}>500 \mathrm{GeV}$, a large $\eta$ separation, $\left|\Delta \eta_{j j}\right|>2.5$, and $\max \left(z_{\ell}^{*}\right)<0.75$, where $z_{\ell}^{*}=$ $\left|\eta_{\ell}-\left(\eta_{j 1}+\eta_{j 2}\right) / 2\right| /\left|\Delta \eta_{j j}\right|$ is the Zeppenfeld variable [25], $\eta_{\ell}$ is the pseudorapidity of a lepton, and $\eta_{j 1}$ and $\eta_{j 2}$ are the pseudorapidities of the leading and subleading jet, respectively.

Techniques for identification of $b$ quark jets are used to veto top quark events. These techniques are based on $b$ quark jet tagging criteria that combine the information from displaced tracks with the information from secondary vertices associated with the jet using a multivariate technique, and on the possible presence of a soft muon in the event from the semileptonic decay of the bottom quark $[26,27]$. A minimum dilepton mass, $m_{\ell \ell}>20 \mathrm{GeV}$, is required to reduce nonprompt lepton processes. To reduce the background from $W Z$ production, events with a third loosely identified lepton with $p_{\mathrm{T}}>10 \mathrm{GeV}$ or an identified hadronically decaying $\tau$ lepton with $p_{\mathrm{T}}>18 \mathrm{GeV}$ are rejected. Drell-Yan events can be selected if the charge of one lepton is measured incorrectly. The charge mismeasurement in dimuon events is negligible, while this 
background is not negligible for dielectron events. An invariant mass veto, $\left|m_{\ell \ell}-m_{\mathrm{Z}}\right|>15 \mathrm{GeV}$, is imposed for $e^{ \pm} e^{ \pm}$events. The Drell-Yan background is further reduced by requiring $p_{\mathrm{T}}^{\text {miss }}>40 \mathrm{GeV}$.

A $W Z \rightarrow 3 e \nu$ control region is defined by requiring an additional identified lepton with $p_{\mathrm{T}}>10 \mathrm{GeV}$ and an opposite-sign same-flavor lepton pair with an invariant mass consistent with that of the $Z$ boson. The background contribution from charge misidentification is estimated by applying a data-to-simulation efficiency correction to charge misidentified electrons in bins of $\eta$. The charge misidentification rate, estimated using Drell-Yan events, is between about $0.01 \%$ in the barrel region and about $0.3 \%$ in the end cap region for electrons.

The nonprompt lepton backgrounds originating from leptonic decays of heavy quarks, hadrons misidentified as leptons, and electrons from photon conversions are suppressed by the identification and isolation requirements imposed on electrons and muons. The remaining contribution from the nonprompt lepton background is estimated directly from data following the technique described in Ref. [11]. All other background processes are estimated from simulation applying corrections to account for small differences between data and simulation, as described below.

The lepton trigger, reconstruction, and selection efficiencies are measured using Drell-Yan events that provide an unbiased sample with high purity. The estimated uncertainty is less than $2 \%$ per lepton. The jet energy scale and resolution uncertainties give rise to an uncertainty in the yields of up to $7 \%$. The uncertainty in the estimated event yields related to the top quark veto is evaluated by using a $Z / \gamma^{*} \rightarrow \ell^{+} \ell^{-}$ sample with at least two reconstructed jets and is $3 \%$ or smaller. The statistical uncertainty due to the finite size of each simulated sample is also taken into account. The uncertainty of $2.5 \%$ in the integrated luminosity determination [28] is considered for all processes estimated from simulation and for the fiducial cross section. The normalization of the processes with misidentified leptons is estimated with a systematic uncertainty of $30 \%$. The $W Z$ background normalization uncertainty is $20 \%-40 \%$, dominated by the statistical uncertainty arising from the small number of events in the trilepton control region. Theoretical uncertainties are estimated by varying simultaneously the renormalization and factorization scales up and down by a factor of 2 from their nominal value in each event, and, depending on kinematic region, are up to $12 \%$ for the signal normalization and $20 \%$ for the triboson background normalization. The interference between the EW signal and the QCD-induced same-sign $W$ boson production background is estimated using the PHANTOM 1.2.8 generator [29] and is treated as a systematic uncertainty of $4.5 \%$ in the signal yield. An uncertainty in the parton distribution function contributes $5 \%$ to the signal times acceptance [30].

The simulated signal and background yields, as well as the observed data yields, are shown in Table I. See
TABLE I. Estimated signal and background yields after the selection. The statistical and systematic uncertainties are added in quadrature. The processes contributing to less than $1 \%$ of the total background are not listed, but included in the total background yield.

\begin{tabular}{lc}
\hline \hline Data & 201 \\
\hline Signal + total background & $205 \pm 13$ \\
Signal & $66.9 \pm 2.4$ \\
Total background & $138 \pm 13$ \\
Nonprompt & $88 \pm 13$ \\
$W Z$ & $25.1 \pm 1.1$ \\
QCD $W W$ & $4.8 \pm 0.4$ \\
$W \gamma$ & $8.3 \pm 1.6$ \\
Triboson & $5.8 \pm 0.8$ \\
Wrong sign & $5.2 \pm 1.1$ \\
\hline \hline
\end{tabular}

Supplemental Material [31], which includes Ref. [32] for a table with more detailed results. The two dominant sources of background events arise from nonprompt leptons and the $W Z$ process. The distributions of $m_{j j}$ and $m_{\ell \ell}$ in the signal region are shown in Fig. 2. An excess of events with respect to the background-only hypothesis is observed. In order to quantify the significance of the observation of the EW production of same-sign $W$ boson pairs, a statistical analysis of the event yields is performed with a fit to the $\left(m_{j j}, m_{\ell \ell}\right)$ two-dimensional distributions. The fit is performed simultaneously in the signal region and in the $W Z$ control region, although only the $m_{j j}$ distribution is used in the latter region. The aim of using the $W Z$ control region is to determine the number of $W Z$ background events in the signal region as a function of $m_{j j}$. The lepton flavor is not used to separate event samples. The EW signal yield and the $W Z$ background normalization are free parameters of the fit. All background contributions can vary within the estimated uncertainties. The data excess is quantified by calculating the $p$ value using a profile likelihood ratio test statistic [33-35]. The observed (expected) statistical significance of the signal is 5.5 (5.7) standard deviations. The ratio of measured signal event yield to that expected from the $\mathrm{SM}$ is $0.90 \pm 0.22$.

The cross section is extracted in a fiducial signal region, defined using $\mathrm{MC}$ generator quantities by requiring two same-sign leptons from $W$ boson decays with $p_{\mathrm{T}}^{\ell}>20 \mathrm{GeV}$ and $\left|\eta_{\ell}\right|<2.5$, two jets with $p_{\mathrm{T}}^{j}>30 \mathrm{GeV}$ and $\left|\eta^{j}\right|<5.0$, $m_{j j}>500 \mathrm{GeV}$, and $\left|\Delta \eta_{j j}\right|>2.5$. In this definition, the leptons are defined at particle level postfinal state radiation and $W \rightarrow \tau \nu \rightarrow \ell \nu \nu \nu$ decays are excluded. The measured cross section is corrected for the acceptance in this region using the MADGRAPH5_aMC@NLO generator, which is also used to estimate the theoretical cross section at LO. The fiducial cross section is measured to be $\sigma_{\text {fid }}\left(W^{ \pm} W^{ \pm} j j\right)=$ $3.83 \pm 0.66$ (stat) \pm 0.35 (syst) fb. The predicted theoretical cross section at LO is $4.25 \pm 0.27 \mathrm{fb}$, in agreement with the measurement. The uncertainty in the theoretical cross 

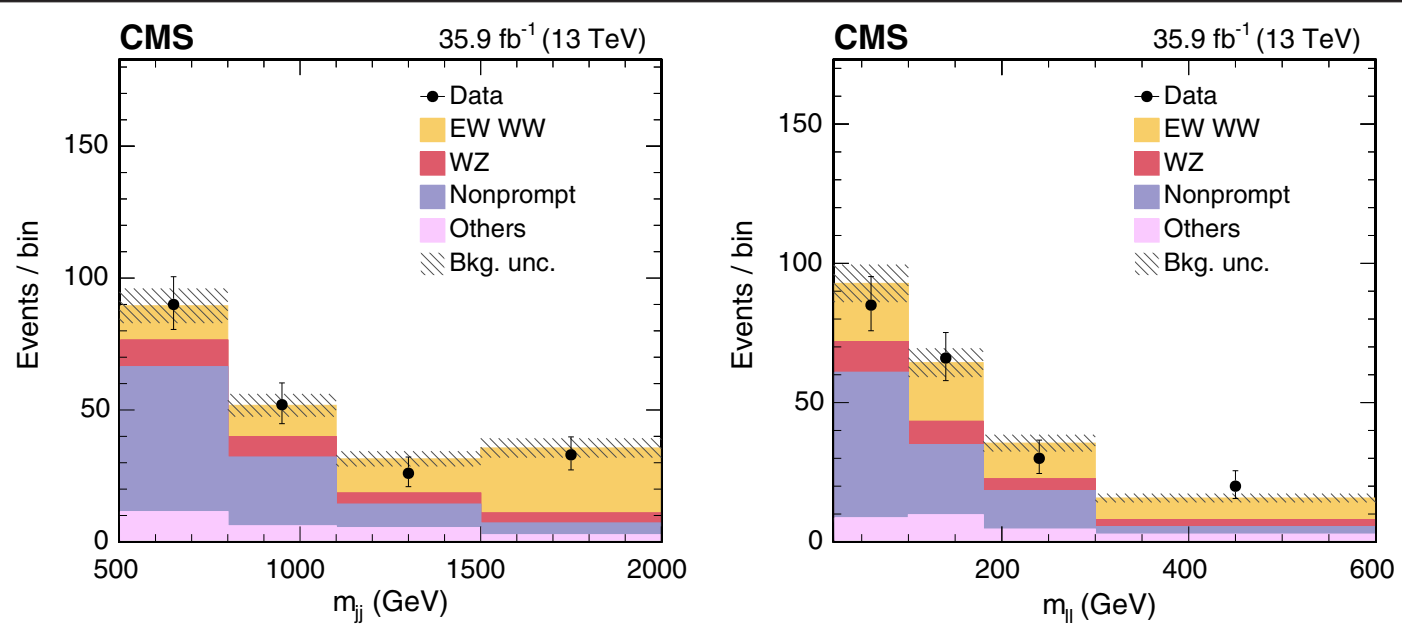

FIG. 2. Distributions of $m_{j j}$ (left) and $m_{\ell \ell}$ (right) in the signal region. The normalization of the EW $W^{ \pm} W^{ \pm}$and background distributions corresponds to the result of the fit. The hatched bands include statistical and systematic uncertainties from the predicted yields. The histograms for other backgrounds include the contributions from QCD $W W, W \gamma$, wrong-sign events, double parton scattering, and triboson processes. The overflow is included in the last bin.

section stems from scale variations and parton distribution functions. Complete NLO QCD and EW corrections to $W^{+} W^{+}$scattering [36] are computed using similar selection requirements as presented in this paper. The NLO EW corrections to the fiducial cross section are dominant and negative $(-13 \%)$. The overall efficiency within the fiducial region is $34.8 \pm 0.3$ (stat) \pm 2.3 (syst) $\%$.

Various extensions of the SM alter the couplings between vector bosons. Reference [6] proposes nine independent charge conjugate and parity-conserving dimension- 8 effective operators to modify the quartic couplings. In this case, the $m_{\ell \ell}$ distributions in both the signal and $W Z$ regions are used to perform the statistical analysis. The EW production is treated as a background consistent with the SM expectation and can vary within the estimated uncertainties. The observed and expected 95\% confidence level (C.L.) limits for the nine coefficients, shown in Table II, are obtained by varying the effective operators one by one. The effect of possible AQGCs on the $W Z$ process in the signal region is negligible because the background is normalized using data.

TABLE II. Observed and expected 95\% C.L. limits on the coefficients for higher-order (dimension-8) operators in the effective field theory Lagrangian.

\begin{tabular}{lcc}
\hline \hline & Observed limits $\left(\mathrm{TeV}^{-4}\right)$ & Expected limits $\left(\mathrm{TeV}^{-4}\right)$ \\
\hline $\mathrm{f}_{\mathrm{S} 0} / \Lambda^{4}$ & {$[-7.7,7.7]$} & {$[-7.0,7.2]$} \\
$\mathrm{f}_{\mathrm{S} 1} / \Lambda^{4}$ & {$[-21.6,21.8]$} & {$[-19.9,20.2]$} \\
$\mathrm{f}_{\mathrm{M} 0} / \Lambda^{4}$ & {$[-6.0,5.9]$} & {$[-5.6,5.5]$} \\
$\mathrm{f}_{\mathrm{M} 1} / \Lambda^{4}$ & {$[-8.7,9.1]$} & {$[-7.9,8.5]$} \\
$\mathrm{f}_{\mathrm{M} 6} / \Lambda^{4}$ & {$[-11.9,11.8]$} & {$[-11.1,11.0]$} \\
$\mathrm{f}_{\mathrm{M} 7} / \Lambda^{4}$ & {$[-13.3,12.9]$} & {$[-12.4,11.8]$} \\
$\mathrm{f}_{\mathrm{T} 0} / \Lambda^{4}$ & {$[-0.62,0.65]$} & {$[-0.58,0.61]$} \\
$\mathrm{f}_{\mathrm{T} 1} / \Lambda^{4}$ & {$[-0.28,0.31]$} & {$[-0.26,0.29]$} \\
$\mathrm{f}_{\mathrm{T} 2} / \Lambda^{4}$ & {$[-0.89,1.02]$} & {$[-0.80,0.95]$} \\
\hline \hline
\end{tabular}

The table also shows the most stringent 95\% C.L. limits reported by the CMS Collaboration previously.

Doubly charged Higgs bosons are predicted in models that contain a Higgs triplet field. Some of these scenarios predict same-sign lepton events from $W^{ \pm} W^{ \pm}$decays with a VBF topology. The Georgi-Machacek model of Higgs triplets [37] is considered. The couplings depend on $m_{H^{ \pm \pm}}$and the parameter $\sin \theta_{H}$, or $s_{H}$, where $s_{H}^{2}$ denotes the fraction of the $W$ boson mass generated by the vacuum expectation value of the triplets. The expected signal event yields for VBF production of $H^{ \pm \pm}$decaying to $W^{ \pm} W^{ \pm}$are directly proportional to $s_{H}^{2}$. The remaining five parameters in the model are adjusted to achieve the given $m_{H^{ \pm \pm}}$ hypothesis, while requiring one of the scalar singlets to have a mass of $125 \mathrm{GeV}$. By using the $\left(m_{j j}, m_{\ell \ell}\right)$ twodimensional distribution in the signal region and the $m_{j j}$ distribution in the $W Z$ control region simultaneously to discriminate between signal and background processes, $95 \%$ C.L. upper limits on $\sigma_{\mathrm{VBF}}\left(H^{ \pm \pm}\right) \mathcal{B}\left(H^{ \pm \pm} \rightarrow W^{ \pm} W^{ \pm}\right)$ can be derived, as shown in Fig. 3. The observed limit excludes $s_{H}$ values greater than 0.18 and 0.44 at $m\left(H^{ \pm \pm}\right)=200$ and $1000 \mathrm{GeV}$, respectively. See Supplemental Material [31] for the expected and observed 95\% C.L. upper limits on $s_{H}$ in the Georgi-Machacek model as a function of doubly charged Higgs boson mass.

In summary, we present the first observation of electroweak production of same-sign $W$ boson pairs in protonproton collisions. The data sample corresponds to an integrated luminosity of $35.9 \mathrm{fb}^{-1}$ collected at $\sqrt{s}=$ $13 \mathrm{TeV}$ with the CMS detector. Events are selected by requiring exactly two leptons of the same charge, moderate $p_{\mathrm{T}}^{\text {miss }}$, and two jets with large rapidity separation and large dijet mass. The two main background processes after the event selection has been applied are nonprompt lepton and $W Z \rightarrow 3 \ell \nu$ processes. The observed significance is 


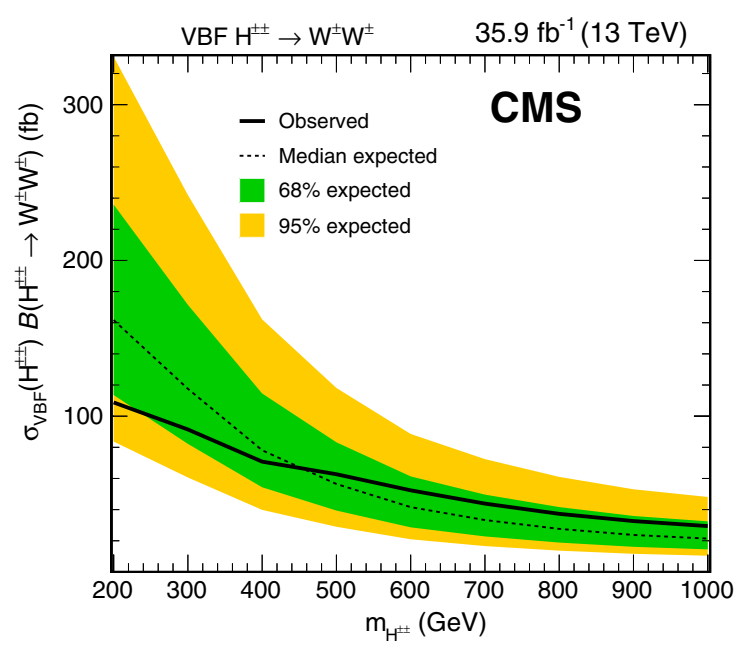

FIG. 3. Expected and observed $95 \%$ C.L. upper limits on the cross section times branching fraction, $\sigma_{\mathrm{VBF}}\left(H^{ \pm \pm}\right) \mathcal{B}\left(H^{ \pm \pm} \rightarrow\right.$ $W^{ \pm} W^{ \pm}$) as a function of doubly charged Higgs boson mass.

5.5 standard deviations, where a significance of 5.7 standard deviations is expected based on the SM. The ratio of measured event yields to that expected from the standard model at leading order is $0.90 \pm 0.22$. A cross section measurement in a fiducial region is reported consistent with SM predictions. Bounds on the structure of quartic vector boson interactions are improved by a factor of up to 6 compared to previous results. Upper limits are given on the production cross section times branching fraction of doubly charged Higgs bosons.

We congratulate our colleagues in the CERN accelerator departments for the excellent performance of the LHC and thank the technical and administrative staffs at CERN and at other CMS institutes for their contributions to the success of the CMS effort. In addition, we gratefully acknowledge the computing centers and personnel of the Worldwide LHC Computing Grid for delivering so effectively the computing infrastructure essential to our analyses. Finally, we acknowledge the enduring support for the construction and operation of the LHC and the CMS detector provided by the following funding agencies: BMWFW and FWF (Austria); FNRS and FWO (Belgium); CNPq, CAPES, FAPERJ, and FAPESP (Brazil); MES (Bulgaria); CERN; CAS, MoST, and NSFC (China); COLCIENCIAS (Colombia); MSES and CSF (Croatia); RPF (Cyprus); SENESCYT (Ecuador); MoER, ERC IUT, and ERDF (Estonia); Academy of Finland, MEC, and HIP (Finland); CEA and CNRS/IN2P3 (France); BMBF, DFG, and HGF (Germany); GSRT (Greece); OTKA and NIH (Hungary); DAE and DST (India); IPM (Iran); SFI (Ireland); INFN (Italy); MSIP and NRF (Republic of Korea); LAS (Lithuania); MOE and UM (Malaysia); BUAP, CINVESTAV, CONACYT, LNS, SEP, and UASLP-FAI (Mexico); MBIE (New Zealand); PAEC (Pakistan); MSHE and NSC (Poland); FCT (Portugal); JINR (Dubna); MON, RosAtom, RAS, RFBR and RAEP (Russia); MESTD (Serbia); SEIDI, CPAN, PCTI and FEDER (Spain); Swiss Funding
Agencies (Switzerland); MST (Taipei); ThEPCenter, IPST, STAR, and NSTDA (Thailand); TUBITAK and TAEK (Turkey); NASU and SFFR (Ukraine); STFC (United Kingdom); DOE and NSF (USA).

[1] ATLAS Collaboration, Observation of a new particle in the search for the standard model Higgs boson with the ATLAS detector at the LHC, Phys. Lett. B 716, 1 (2012).

[2] CMS Collaboration, Observation of a new boson at a mass of $125 \mathrm{GeV}$ with the CMS experiment at the LHC, Phys. Lett. B 716, 30 (2012).

[3] CMS Collaboration, Observation of a new boson with mass near $125 \mathrm{GeV}$ in $p p$ collisions at $\sqrt{s}=7$ and $8 \mathrm{TeV}$, J. High Energy Phys. 06 (2013) 081.

[4] D. Espriu and B. Yencho, Longitudinal $W W$ scattering in light of the Higgs boson discovery, Phys. Rev. D 87, 055017 (2013).

[5] J. Chang, K. Cheung, Ch.-T. Lu, and T.-Ch. Yuan, WW scattering in the era of post-Higgs-boson discovery, Phys. Rev. D 87, 093005 (2013).

[6] O. J. P. Éboli, M. C. Gonzalez-Garcia, and J. K. Mizukoshi, $p p \rightarrow j j e^{ \pm} \mu^{ \pm} \nu \nu$ and $j j e^{\mp} \mu^{ \pm} \nu \nu$ at $\mathcal{O}\left(\alpha_{e m}^{6}\right)$ and $\mathcal{O}\left(\alpha_{e m}^{4} \alpha_{s}^{2}\right)$ for the study of the quartic electroweak gauge boson vertex at CERN LHC, Phys. Rev. D 74, 073005 (2006).

[7] Ch. Englert, E. Re, and M. Spannowsky, Triplet Higgs boson collider phenomenology after the LHC, Phys. Rev. D 87, 095014 (2013).

[8] Ch. Englert, E. Re, and M. Spannowsky, Pinning down Higgs triplets at the LHC, Phys. Rev. D 88, 035024 (2013).

[9] Ch.-W. Chiang, T. Nomura, and K. Tsumura, Search for doubly charged Higgs bosons using the same-sign diboson mode at the LHC, Phys. Rev. D 85, 095023 (2012).

[10] ATLAS Collaboration, Evidence for Electroweak Production of $W^{ \pm} W^{ \pm} j j$ in $p p$ Collisions at $\sqrt{s}=8 \mathrm{TeV}$ with the ATLAS Detector, Phys. Rev. Lett. 113, 141803 (2014).

[11] CMS Collaboration, Study of Vector Boson Scattering and Search for New Physics in Events with Two Same-Sign Leptons and Two Jets, Phys. Rev. Lett. 114, 051801 (2015).

[12] CMS Collaboration, The CMS experiment at the CERN LHC, J. Instrum. 3, S08004 (2008).

[13] J. Alwall, R. Frederix, S. Frixione, V. Hirschi, F. Maltoni, O. Mattelaer, H.-S. Shao, T. Stelzer, P. Torrielli, and M. Zaro, The automated computation of tree-level and next-to-leading order differential cross sections, and their matching to parton shower simulations, J. High Energy Phys. 07 (2014) 079.

[14] T. Sjöstrand, S. Ask, J. R. Christiansen, R. Corke, N. Desai, P. Ilten, S. Mrenna, S. Prestel, C. O. Rasmussen, and P.Z. Skands, An introduction to PYTHIA 8.2, Comput. Phys. Commun. 191, 159 (2015).

[15] P. Skands, S. Carrazza, and J. Rojo, Tuning PYTHIA 8.1: The Monash 2013 tune, Eur. Phys. J. C 74, 3024 (2014).

[16] CMS Collaboration, Event generator tunes obtained from underlying event and multiparton scattering measurements, Eur. Phys. J. C 76, 155 (2016).

[17] R. D. Ball, V. Bertone, S. Carrazza, Ch. S. Deans, L. Del Debbio, S. Forte, A. Guffanti, N. P. Hartland, J. I. Latorre, J. Rojo, and M. Ubiali (NNPDF), Parton distributions for the LHC Run II, J. High Energy Phys. 04 (2015) 040. 
[18] S. Agostinelli et al. (GEANT4 Collaboration), GEANT4A simulation toolkit, Nucl. Instrum. Methods Phys. Res., Sect. A 506, 250 (2003).

[19] CMS Collaboration, The CMS trigger system, J. Instrum. 12, P01020 (2017).

[20] CMS Collaboration, Particle-flow reconstruction and global event description with the CMS detector, J. Instrum. 12, P10003 (2017).

[21] CMS Collaboration, Performance of electron reconstruction and selection with the CMS detector in proton-proton collisions at $\sqrt{s}=8 \mathrm{TeV}$, J. Instrum. 10, P06005 (2015).

[22] M. Cacciari, G. P. Salam, and G. Soyez, The anti- $k_{t}$ jet clustering algorithm, J. High Energy Phys. 04 (2008) 063.

[23] M. Cacciari, G. P. Salam, and G. Soyez, FastJet user manual, Eur. Phys. J. C 72, 1896 (2012).

[24] M. Cacciari and G. P. Salam, Dispelling the $N^{3}$ myth for the $k_{t}$ jet finder, Phys. Lett. B 641, 57 (2006).

[25] D. L. Rainwater, R. Szalapski, and D. Zeppenfeld, Probing color singlet exchange in $Z+$ two jet events at the CERN LHC, Phys. Rev. D 54, 6680 (1996).

[26] CMS Collaboration, Identification of b-quark jets with the CMS experiment, J. Instrum. 8, P04013 (2013).

[27] CMS Collaboration, CMS Report No. CMS-PAS-BTV-15001, 2016, http://cds.cern.ch/record/2138504.

[28] CMS Collaboration, CMS Report No. CMS-PAS-LUM-17001, 2017, http://cds.cern.ch/record/2257069.
[29] A. Ballestrero, A. Belhouari, G. Bevilacqua, V. Kashkan, and E. Maina, PHANTOM: A Monte Carlo event generator for six parton final states at high energy colliders, Comput. Phys. Commun. 180, 401 (2009).

[30] J. Butterworth et al., PDF4LHC recommendations for LHC Run II, J. Phys. G 43, 023001 (2016).

[31] See Supplemental Material http://link.aps.org/supplemental/ 10.1103/PhysRevLett.120.081801 for the estimated signal and background yields after selection; expected and observed $95 \%$ C.L. upper limits on $s_{H}$ in the Georgi-Machacek model as a function of doubly charged Higgs boson mass.

[32] M. Zaro and H. Logan, CERN Report No. LHCHXSWG2015-001, 2015.

[33] T. Junk, Confidence level computation for combining searches with small statistics, Nucl. Instrum. Methods Phys. Res., Sect. A 434, 435 (1999).

[34] A. L. Read, Presentation of search results: The $C L_{s}$ technique, J. Phys. G 28, 2693 (2002).

[35] G. Cowan, K. Cranmer, E. Gross, and O. Vitells, Asymptotic formulas for likelihood-based tests of new physics, Eur. Phys. J. C 71, 1554 (2011); Erratum, Eur. Phys. J. C 73, 2501 (2013).

[36] B. Biedermann, A. Denner, and M. Pellen, Complete NLO corrections to $\mathrm{W}^{+} \mathrm{W}^{+}$scattering and its irreducible background at the LHC, J. High Energy Phys. 10 (2017) 124.

[37] H. Georgi and M. Machacek, Doubly charged Higgs bosons, Nucl. Phys. B262, 463 (1985).

A. M. Sirunyan, ${ }^{1}$ A. Tumasyan, ${ }^{1}$ W. Adam, ${ }^{2}$ F. Ambrogi, ${ }^{2}$ E. Asilar, ${ }^{2}$ T. Bergauer, ${ }^{2}$ J. Brandstetter, ${ }^{2}$ E. Brondolin, ${ }^{2}$ M. Dragicevic, ${ }^{2}$ J. Erö, ${ }^{2}$ M. Flechl, ${ }^{2}$ M. Friedl, ${ }^{2}$ R. Frühwirth, ${ }^{2, b}$ V. M. Ghete, ${ }^{2}$ J. Grossmann, ${ }^{2}$ J. Hrubec, ${ }^{2}$ M. Jeitler, ${ }^{2, b}$ A. König, ${ }^{2}$ N. Krammer, ${ }^{2}$ I. Krätschmer, ${ }^{2}$ D. Liko, ${ }^{2}$ T. Madlener, ${ }^{2}$ I. Mikulec, ${ }^{2}$ E. Pree, ${ }^{2}$ N. Rad, ${ }^{2}$ H. Rohringer, ${ }^{2}$ J. Schieck, ${ }^{2, b}$ R. Schöfbeck, ${ }^{2}$ M. Spanring, ${ }^{2}$ D. Spitzbart, ${ }^{2}$ W. Waltenberger, ${ }^{2}$ J. Wittmann, ${ }^{2}$ C.-E. Wulz, ${ }^{2, b}$ M. Zarucki, ${ }^{2}$ V. Chekhovsky, ${ }^{3}$ V. Mossolov, ${ }^{3}$ J. Suarez Gonzalez, ${ }^{3}$ E. A. De Wolf, ${ }^{4}$ D. Di Croce, ${ }^{4}$ X. Janssen, ${ }^{4}$ J. Lauwers, ${ }^{4}$

M. Van De Klundert, ${ }^{4}$ H. Van Haevermaet, ${ }^{4}$ P. Van Mechelen, ${ }^{4}$ N. Van Remortel, ${ }^{4}$ S. Abu Zeid, ${ }^{5}$ F. Blekman, ${ }^{5}$ J. D'Hondt, ${ }^{5}$ I. De Bruyn, ${ }^{5}$ J. De Clercq, ${ }^{5}$ K. Deroover, ${ }^{5}$ G. Flouris, ${ }^{5}$ D. Lontkovskyi, ${ }^{5}$ S. Lowette, ${ }^{5}$ S. Moortgat, ${ }^{5}$ L. Moreels, ${ }^{5}$ Q. Python, ${ }^{5}$ K. Skovpen, ${ }^{5}$ S. Tavernier, ${ }^{5}$ W. Van Doninck, ${ }^{5}$ P. Van Mulders, ${ }^{5}$ I. Van Parijs, ${ }^{5}$ D. Beghin, ${ }^{6}$ H. Brun, ${ }^{6}$ B. Clerbaux, ${ }^{6}$ G. De Lentdecker, ${ }^{6}$ H. Delannoy, ${ }_{6}^{6}$ B. Dorney, ${ }^{6}$ G. Fasanella, ${ }^{6}$ L. Favart, ${ }^{6}$ R. Goldouzian, ${ }^{6}$ A. Grebenyuk, ${ }^{6}$ G. Karapostoli, ${ }^{6}$ T. Lenzi, ${ }^{6}$ J. Luetic, ${ }^{6}$ T. Maerschalk, ${ }^{6}$ A. Marinov, ${ }^{6}$ A. Randle-conde, ${ }^{6}$ T. Seva, ${ }^{6}$ E. Starling, ${ }^{6}$ C. Vander Velde, ${ }^{6}$ P. Vanlaer, ${ }^{6}$ D. Vannerom, ${ }^{6}$ R. Yonamine, ${ }^{6}$ F. Zenoni, ${ }^{6}$ F. Zhang, ${ }^{6, c}$ A. Cimmino, ${ }^{7}$ T. Cornelis, ${ }^{7}$ D. Dobur, ${ }^{7}$ A. Fagot, ${ }^{7}$ M. Gul, ${ }^{7}$ I. Khvastunov, ${ }^{7, d}$ D. Poyraz, ${ }^{7}$ C. Roskas, ${ }^{7}$ S. Salva, ${ }^{7}$ M. Tytgat, ${ }^{7}$ W. Verbeke, ${ }^{7}$ N. Zaganidis, ${ }^{7}$ H. Bakhshiansohi, ${ }^{8}$ O. Bondu, ${ }^{8}$ S. Brochet, ${ }^{8}$ G. Bruno, ${ }^{8}$ C. Caputo, ${ }^{8}$ A. Caudron, ${ }^{8}$ P. David, ${ }^{8}$ S. De Visscher, ${ }^{8}$ C. Delaere, ${ }^{8}$ M. Delcourt, ${ }^{8}$ B. Francois, ${ }^{8}$ A. Giammanco,${ }^{8}$ M. Komm, ${ }^{8}$ G. Krintiras, ${ }^{8}$ V. Lemaitre, ${ }^{8}$ A. Magitteri, ${ }^{8}$ A. Mertens, ${ }^{8}$ M. Musich, ${ }^{8}$ K. Piotrzkowski, ${ }^{8}$ L. Quertenmont,${ }^{8}$ A. Saggio, ${ }^{8}$ M. Vidal Marono, ${ }^{8}$ S. Wertz, ${ }^{8}$ J. Zobec, ${ }^{8}$ N. Beliy, ${ }^{9}$ W. L. Aldá Júnior, ${ }^{10}$ F. L. Alves,${ }^{10}$ G. A. Alves, ${ }^{10}$ L. Brito, ${ }^{10}$ M. Correa Martins Junior, ${ }^{10}$ C. Hensel,${ }^{10}$ A. Moraes, ${ }^{10}$ M. E. Pol,${ }^{10}$ P. Rebello Teles,${ }^{10}$ E. Belchior Batista Das Chagas, ${ }^{11}$ W. Carvalho, ${ }^{11}$ J. Chinellato,${ }^{11, e}$ E. Coelho, ${ }^{11}$ E. M. Da Costa, ${ }^{11}$ G. G. Da Silveira,${ }^{11, f}$ D. De Jesus Damiao, ${ }^{11}$ S. Fonseca De Souza, ${ }^{11}$ L. M. Huertas Guativa, ${ }^{11}$ H. Malbouisson, ${ }^{11}$ M. Melo De Almeida,${ }^{11}$ C. Mora Herrera, ${ }^{11}$ L. Mundim,${ }^{11}$ H. Nogima, ${ }^{11}$ L. J. Sanchez Rosas, ${ }^{11}$ A. Santoro, ${ }^{11}$ A. Sznajder, ${ }^{11}$ M. Thiel, ${ }^{11}$ E. J. Tonelli Manganote, ${ }^{11, e}$ F. Torres Da Silva De Araujo, ${ }^{11}$ A. Vilela Pereira, ${ }^{11}$ S. Ahuja, ${ }^{12 a}$ C. A. Bernardes, ${ }^{12 a}$ T. R. Fernandez Perez Tomei, ${ }^{12 a}$ E. M. Gregores, ${ }^{12 b}$ P. G. Mercadante, ${ }^{12 b}$ S. F. Novaes, ${ }^{12 a}$ Sandra S. Padula, ${ }^{12 \mathrm{a}}$ D. Romero Abad, ${ }^{12 \mathrm{~b}}$ J. C. Ruiz Vargas, ${ }^{12 \mathrm{a}}$ A. Aleksandrov,${ }^{13}$ R. Hadjiiska, ${ }^{13}$ P. Iaydjiev, ${ }^{13}$ M. Misheva, ${ }^{13}$ M. Rodozov, ${ }^{13}$ M. Shopova, ${ }^{13}$ G. Sultanov, ${ }^{13}$ A. Dimitrov, ${ }^{14}$ I. Glushkov,${ }^{14}$ L. Litov, ${ }^{14}$ B. Pavlov, ${ }^{14}$ P. Petkov, ${ }^{14}$ W. Fang, ${ }^{15, g}$ X. Gao, ${ }^{15, g}$ L. Yuan, ${ }^{15}$ M. Ahmad, ${ }^{16}$ J. G. Bian, ${ }^{16}$ G. M. Chen, ${ }^{16}$ H. S. Chen, ${ }^{16}$ M. Chen, ${ }^{16}$ 
Y. Chen, ${ }^{16}$ C. H. Jiang, ${ }^{16}$ D. Leggat, ${ }^{16}$ H. Liao, ${ }^{16}$ Z. Liu, ${ }^{16}$ F. Romeo, ${ }^{16}$ S. M. Shaheen, ${ }^{16}$ A. Spiezia, ${ }^{16}$ J. Tao, ${ }^{16}$ C. Wang, ${ }^{16}$ Z. Wang, ${ }^{16}$ E. Yazgan, ${ }^{16}$ H. Zhang, ${ }^{16}$ S. Zhang, ${ }^{16}$ J. Zhao, ${ }^{16}$ Y. Ban,${ }^{17}$ G. Chen,${ }^{17}$ Q. Li, ${ }^{17}$ S. Liu, ${ }^{17}$ Y. Mao, ${ }^{17}$ S. J. Qian, ${ }^{17}$ D. Wang, ${ }^{17}$ Z. Xu,${ }^{17}$ C. Avila, ${ }^{18}$ A. Cabrera, ${ }^{18}$ L. F. Chaparro Sierra, ${ }^{18}$ C. Florez, ${ }^{18}$ C. F. González Hernández, ${ }^{18}$ J. D. Ruiz Alvarez, ${ }^{18}$ B. Courbon, ${ }^{19}$ N. Godinovic, ${ }^{19}$ D. Lelas, ${ }^{19}$ I. Puljak, ${ }^{19}$ P. M. Ribeiro Cipriano, ${ }^{19}$ T. Sculac, ${ }^{19}$ Z. Antunovic, ${ }^{20}$ M. Kovac, ${ }^{20}$ V. Brigljevic, ${ }^{21}$ D. Ferencek, ${ }^{21}$ K. Kadija, ${ }^{21}$ B. Mesic, ${ }^{21}$ A. Starodumov, ${ }^{21, \mathrm{~h}}$ T. Susa, ${ }^{21}$

M. W. Ather, ${ }^{22}$ A. Attikis, ${ }^{22}$ G. Mavromanolakis, ${ }^{22}$ J. Mousa, ${ }^{22}$ C. Nicolaou, ${ }^{22}$ F. Ptochos, ${ }^{22}$ P. A. Razis,${ }^{22}$ H. Rykaczewski, ${ }^{22}$ M. Finger, ${ }^{23, i}$ M. Finger Jr., ${ }^{23, i}$ E. Carrera Jarrin, ${ }^{24}$ A. A. Abdelalim, ${ }^{25, j, k}$ Y. Mohammed, ${ }^{25,1}$ E. Salama, ${ }^{25, \mathrm{~m}, \mathrm{n}}$ R. K. Dewanjee, ${ }^{26}$ M. Kadastik,${ }^{26}$ L. Perrini, ${ }^{26}$ M. Raidal, ${ }^{26}$ A. Tiko, ${ }^{26}$ C. Veelken, ${ }^{26}$ P. Eerola, ${ }^{27}$ H. Kirschenmann, ${ }^{27}$ J. Pekkanen, ${ }^{27}$ M. Voutilainen, ${ }^{27}$ J. Havukainen, ${ }^{28}$ J. K. Heikkilä, ${ }^{28}$ T. Järvinen, ${ }^{28}$ V. Karimäki, ${ }^{28}$ R. Kinnunen, ${ }^{28}$ T. Lampén, ${ }^{28}$ K. Lassila-Perini, ${ }^{28}$ S. Laurila, ${ }^{28}$ S. Lehti, ${ }^{28}$ T. Lindén, ${ }^{28}$ P. Luukka, ${ }^{28}$ H. Siikonen, ${ }^{28}$ E. Tuominen, ${ }^{28}$ J. Tuominiemi, ${ }^{28}$ J. Talvitie, ${ }^{29}$ T. Tuuva, ${ }^{29}$ M. Besancon, ${ }^{30}$ F. Couderc, ${ }^{30}$ M. Dejardin, ${ }^{30}$ D. Denegri, ${ }^{30}$ J. L. Faure ${ }^{30}$ F. Ferri, ${ }^{30}$ S. Ganjour, ${ }^{30}$ S. Ghosh, ${ }^{30}$ A. Givernaud, ${ }^{30}$ P. Gras,${ }^{30}$ G. Hamel de Monchenault, ${ }^{30}$ P. Jarry, ${ }^{30}$ I. Kucher, ${ }^{30}$ C. Leloup,${ }^{30}$ E. Locci, ${ }^{30}$ M. Machet, ${ }^{30}$ J. Malcles, ${ }^{30}$ G. Negro, ${ }^{30}$ J. Rander, ${ }^{30}$ A. Rosowsky, ${ }^{30}$ M. Ö. Sahin, ${ }^{30}$ M. Titov,${ }^{30}$ A. Abdulsalam, ${ }^{31}$ C. Amendola ${ }^{31}$ I. Antropov, ${ }^{31}$ S. Baffioni, ${ }^{31}$ F. Beaudette, ${ }^{31}$ P. Busson, ${ }^{31}$ L. Cadamuro, ${ }^{31}$ C. Charlot, ${ }^{31}$ R. Granier de Cassagnac, ${ }^{31}$ M. Jo, ${ }^{31}$ S. Lisniak, ${ }^{31}$ A. Lobanov, ${ }^{31}$ J. Martin Blanco, ${ }^{31}$ M. Nguyen, ${ }^{31}$ C. Ochando, ${ }^{31}$ G. Ortona, ${ }^{31}$ P. Paganini, ${ }^{31}$ P. Pigard, ${ }^{31}$ R. Salerno, ${ }^{31}$ J. B. Sauvan, ${ }^{31}$ Y. Sirois, ${ }^{31}$ A. G. Stahl Leiton, ${ }^{31}$ T. Strebler, ${ }^{31}$ Y. Yilmaz,${ }^{31}$ A. Zabi, ${ }^{31}$ A. Zghiche, ${ }^{31}$ J.-L. Agram, ${ }^{32,0}$ J. Andrea, ${ }^{32}$ D. Bloch, ${ }^{32}$ J.-M. Brom, ${ }^{32}$ M. Buttignol, ${ }^{32}$ E. C. Chabert, ${ }^{32}$ N. Chanon, ${ }^{32}$ C. Collard, ${ }^{32}$ E. Conte, ${ }^{32,0}$ X. Coubez,${ }^{32}$ J.-C. Fontaine, ${ }^{32, o}$ D. Gelé, ${ }^{32}$ U. Goerlach, ${ }^{32}$ M. Jansová, ${ }^{32}$ A.-C. Le Bihan, ${ }^{32}$ N. Tonon, ${ }^{32}$ P. Van Hove, ${ }^{32}$ S. Gadrat,${ }^{33}$ S. Beauceron,,${ }^{34}$ C. Bernet, ${ }^{34}$ G. Boudoul, ${ }^{34}$ R. Chierici, ${ }^{34}$ D. Contardo, ${ }^{34}$ P. Depasse, ${ }^{34}$ H. El Mamouni, ${ }^{34}$ J. Fay,${ }^{34}$ L. Finco, ${ }^{34}$ S. Gascon, ${ }^{34}$ M. Gouzevitch, ${ }^{34}$ G. Grenier, ${ }^{34}$ B. Ille, ${ }^{34}$ F. Lagarde, ${ }^{34}$ I. B. Laktineh,${ }^{34}$ M. Lethuillier, ${ }^{34}$ L. Mirabito, ${ }^{34}$ A. L. Pequegnot, ${ }^{34}$ S. Perries, ${ }^{34}$ A. Popov, ${ }^{34, p}$ V. Sordini, ${ }^{34}$ M. Vander Donckt, ${ }^{34}$ S. Viret,${ }^{34}$ A. Khvedelidze, ${ }^{35, i}$ I. Bagaturia, ${ }^{36, q}$ C. Autermann, ${ }^{37}$ L. Feld, ${ }^{37}$ M. K. Kiesel, ${ }^{37}$ K. Klein, ${ }^{37}$ M. Lipinski, ${ }^{37}$ M. Preuten, ${ }^{37}$ C. Schomakers, ${ }^{37}$ J. Schulz,${ }^{37}$ V. Zhukov, ${ }^{37, p}$ A. Albert, ${ }^{38}$ E. Dietz-Laursonn, ${ }^{38}$ D. Duchardt, ${ }^{38}$ M. Endres, ${ }^{38}$ M. Erdmann, ${ }^{38}$ S. Erdweg, ${ }^{38}$ T. Esch, ${ }^{38}$ R. Fischer, ${ }^{38}$ A. Güth ${ }^{38}$ M. Hamer, ${ }^{38}$ T. Hebbeker, ${ }^{38}$ C. Heidemann, ${ }^{38}$ K. Hoepfner, ${ }^{38}$ S. Knutzen, ${ }^{38}$ M. Merschmeyer, ${ }^{38}$ A. Meyer,${ }^{38}$ P. Millet ${ }^{38}$ S. Mukherjee, ${ }^{38}$ T. Pook, ${ }^{38}$ M. Radziej,${ }^{38}$ H. Reithler, ${ }^{38}$ M. Rieger, ${ }^{38}$ F. Scheuch, ${ }^{38}$ D. Teyssier, ${ }^{38}$ S. Thüer, ${ }^{38}$ G. Flügge,${ }^{39}$ B. Kargoll, ${ }^{39}$ T. Kress, ${ }^{39}$ A. Künsken, ${ }^{39}$ T. Müller,${ }^{39}$ A. Nehrkorn, ${ }^{39}$ A. Nowack, ${ }^{39}$ C. Pistone, ${ }^{39}$ O. Pooth, ${ }^{39}$ A. Stahl,,${ }^{39, r}$ M. Aldaya Martin, ${ }^{40}$ T. Arndt, ${ }^{40}$ C. Asawatangtrakuldee, ${ }^{40}$ K. Beernaert, ${ }^{40}$ O. Behnke, ${ }^{40}$ U. Behrens,${ }^{40}$

\section{A. Bermúdez Martínez, ${ }^{40}$ A. A. Bin Anuar, ${ }^{40}$ K. Borras, ${ }^{40, s}$ V. Botta, ${ }^{40}$ A. Campbell, ${ }^{40}$ P. Connor, ${ }^{40}$}

C. Contreras-Campana, ${ }^{40}$ F. Costanza,${ }^{40}$ C. Diez Pardos,${ }^{40}$ G. Eckerlin, ${ }^{40}$ D. Eckstein, ${ }^{40}$ T. Eichhorn, ${ }^{40}$ E. Eren, ${ }^{40}$

E. Gallo, ${ }^{40, t}$ J. Garay Garcia, ${ }^{40}$ A. Geiser, ${ }^{40}$ A. Gizhko, ${ }^{40}$ J. M. Grados Luyando, ${ }^{40}$ A. Grohsjean, ${ }^{40}$ P. Gunnellini, ${ }^{40}$

M. Guthoff, ${ }^{40}$ A. Harb, ${ }^{40}$ J. Hauk,${ }^{40}$ M. Hempel, ${ }^{40, u}$ H. Jung, ${ }^{40}$ A. Kalogeropoulos, ${ }^{40}$ M. Kasemann, ${ }^{40}$ J. Keaveney, ${ }^{40}$ C. Kleinwort, ${ }^{40}$ I. Korol, ${ }^{40}$ D. Krücker, ${ }^{40}$ W. Lange, ${ }^{40}$ A. Lelek, ${ }^{40}$ T. Lenz,${ }^{40}$ J. Leonard, ${ }^{40}$ K. Lipka, ${ }^{40}$ W. Lohmann, ${ }^{40, u}$ R. Mankel, ${ }^{40}$ I.-A. Melzer-Pellmann, ${ }^{40}$ A. B. Meyer, ${ }^{40}$ G. Mittag, ${ }^{40}$ J. Mnich, ${ }^{40}$ A. Mussgiller, ${ }^{40}$ E. Ntomari, ${ }^{40}$ D. Pitzl, ${ }^{40}$ A. Raspereza, ${ }^{40}$ M. Savitskyi, ${ }^{40}$ P. Saxena, ${ }^{40}$ R. Shevchenko, ${ }^{40}$ S. Spannagel,${ }^{40}$ N. Stefaniuk, ${ }^{40}$ G. P. Van Onsem, ${ }^{40}$

R. Walsh, ${ }^{40}$ Y. Wen, ${ }^{40}$ K. Wichmann, ${ }^{40}$ C. Wissing, ${ }^{40}$ O. Zenaiev, ${ }^{40}$ R. Aggleton, ${ }^{41}$ S. Bein, ${ }^{41}$ V. Blobel,,${ }^{41}$

M. Centis Vignali, ${ }^{41}$ T. Dreyer, ${ }^{41}$ E. Garutti, ${ }^{41}$ D. Gonzalez, ${ }^{41}$ J. Haller, ${ }^{41}$ A. Hinzmann, ${ }^{41}$ M. Hoffmann, ${ }^{41}$ A. Karavdina, ${ }^{41}$

R. Klanner, ${ }^{41}$ R. Kogler, ${ }^{41}$ N. Kovalchuk ${ }^{41}$ S. Kurz, ${ }^{41}$ T. Lapsien, ${ }^{41}$ I. Marchesini, ${ }^{41}$ D. Marconi, ${ }^{41}$ M. Meyer, ${ }^{41}$ M. Niedziela, ${ }^{41}$ D. Nowatschin ${ }^{41}$ F. Pantaleo, ${ }^{41, \mathrm{r}}$ T. Peiffer ${ }^{41}$ A. Perieanu, ${ }^{41}$ C. Scharf,${ }^{41}$ P. Schleper, ${ }^{41}$ A. Schmidt ${ }^{41}$ S. Schumann, ${ }^{41}$ J. Schwandt, ${ }^{41}$ J. Sonneveld, ${ }^{41}$ H. Stadie, ${ }^{41}$ G. Steinbrück, ${ }^{41}$ F. M. Stober, ${ }^{41}$ M. Stöver, ${ }^{41}$ H. Tholen, ${ }^{41}$ D. Troendle, ${ }^{41}$ E. Usai, ${ }^{41}$ A. Vanhoefer ${ }^{41}$ B. Vormwald, ${ }^{41}$ M. Akbiyik, ${ }^{42}$ C. Barth, ${ }^{42}$ M. Baselga, ${ }^{42}$ S. Baur, ${ }^{42}$ E. Butz ${ }^{42}$ R. Caspart, ${ }^{42}$ T. Chwalek, ${ }^{42}$ F. Colombo, ${ }^{42}$ W. De Boer, ${ }^{42}$ A. Dierlamm, ${ }^{42}$ N. Faltermann, ${ }^{42}$ B. Freund, ${ }^{42}$ R. Friese, ${ }^{42}$ M. Giffels, ${ }^{42}$ M. A. Harrendorf, ${ }^{42}$ F. Hartmann, ${ }^{42, \mathrm{r}}$ S. M. Heindl, ${ }^{42}$ U. Husemann, ${ }^{42}$ F. Kassel,${ }^{42, r}$ S. Kudella, ${ }^{42}$ H. Mildner, ${ }^{42}$ M. U. Mozer, ${ }^{42}$ Th. Müller, ${ }^{42}$ M. Plagge, ${ }^{42}$ G. Quast, ${ }^{42}$ K. Rabbertz,${ }^{42}$ M. Schröder,${ }^{42}$ I. Shvetsov, ${ }^{42}$ G. Sieber, ${ }^{42}$ H. J. Simonis, ${ }^{42}$ R. Ulrich, ${ }^{42}$ S. Wayand, ${ }^{42}$ M. Weber, ${ }^{42}$ T. Weiler, ${ }^{42}$ S. Williamson, ${ }^{42}$ C. Wöhrmann, ${ }^{42}$ R. Wolf, ${ }^{42}$ G. Anagnostou, ${ }^{43}$ G. Daskalakis, ${ }^{43}$ T. Geralis, ${ }^{43}$ V. A. Giakoumopoulou, ${ }^{43}$ A. Kyriakis, ${ }^{43}$ D. Loukas, ${ }^{43}$ I. Topsis-Giotis ${ }^{43}$ G. Karathanasis, ${ }^{44}$ S. Kesisoglou, ${ }^{44}$ A. Panagiotou, ${ }^{44}$ N. Saoulidou, ${ }^{44}$ K. Kousouris, ${ }^{45}$ I. Evangelou, ${ }^{46}$ C. Foudas, ${ }^{46}$ P. Kokkas, ${ }^{46}$ S. Mallios, ${ }^{46}$ N. Manthos,${ }^{46}$ I. Papadopoulos, ${ }^{46}$ E. Paradas, ${ }^{46}$ J. Strologas, ${ }^{46}$ F. A. Triantis,${ }^{46}$ M. Csanad,${ }^{47}$ N. Filipovic, ${ }^{47}$ G. Pasztor, ${ }^{47}$ O. Surányi,${ }^{47}$ G. I. Veres, ${ }^{47, v}$ G. Bencze, ${ }^{48}$ C. Hajdu, ${ }^{48}$ D. Horvath, ${ }^{48, w}$ Á. Hunyadi, ${ }^{48}$ F. Sikler, ${ }^{48}$ 
V. Veszpremi, ${ }^{48}$ N. Beni, ${ }^{49}$ S. Czellar, ${ }^{49}$ J. Karancsi, ${ }^{49, x}$ A. Makovec, ${ }^{49}$ J. Molnar, ${ }^{49}$ Z. Szillasi, ${ }^{49}$ M. Bartók, ${ }^{50, v}$ P. Raics,${ }^{50}$ Z. L. Trocsanyi ${ }^{50}$ B. Ujvari, ${ }^{50}$ S. Choudhury,${ }^{51}$ J. R. Komaragiri, ${ }^{51}$ S. Bahinipati, ${ }^{52, y}$ S. Bhowmik, ${ }^{52}$ P. Mal, ${ }^{52}$ K. Mandal, ${ }^{52}$ A. Nayak, ${ }^{52, z}$ D. K. Sahoo, ${ }^{52, y}$ N. Sahoo, ${ }^{52}$ S. K. Swain, ${ }^{52}$ S. Bansal, ${ }^{53}$ S. B. Beri, ${ }^{53}$ V. Bhatnagar, ${ }^{53}$ R. Chawla, ${ }^{53}$ N. Dhingra, ${ }^{53}$ A. K. Kalsi, ${ }^{53}$ A. Kaur, ${ }^{53}$ M. Kaur, ${ }^{53}$ S. Kaur, ${ }^{53}$ R. Kumar, ${ }^{53}$ P. Kumari, ${ }^{53}$ A. Mehta, ${ }^{53}$ J. B. Singh,${ }^{53}$ G. Walia, ${ }^{53}$ Ashok Kumar, ${ }^{54}$ Aashaq Shah, ${ }^{54}$ A. Bhardwaj, ${ }^{54}$ S. Chauhan, ${ }^{54}$ B. C. Choudhary, ${ }^{54}$ R. B. Garg, ${ }^{54}$ S. Keshri, ${ }^{54}$ A. Kumar,${ }^{54}$

S. Malhotra, ${ }^{54}$ M. Naimuddin, ${ }^{54}$ K. Ranjan, ${ }^{54}$ R. Sharma, ${ }^{54}$ R. Bhardwaj, ${ }^{55}$ R. Bhattacharya ${ }^{55}$ S. Bhattacharya, ${ }^{55}$

U. Bhawandeep ${ }^{55}$ S. Dey, ${ }^{55}$ S. Dutt, ${ }^{55}$ S. Dutta ${ }^{55}$ S. Ghosh, ${ }^{55}$ N. Majumdar ${ }^{55}$ A. Modak,${ }^{55}$ K. Mondal,${ }^{55}$ S. Mukhopadhyay, ${ }^{55}$ S. Nandan, ${ }^{55}$ A. Purohit,${ }^{55}$ A. Roy, ${ }^{55}$ D. Roy, ${ }^{55}$ S. Roy Chowdhury, ${ }^{55}$ S. Sarkar, ${ }^{55}$ M. Sharan,${ }^{55}$ S. Thakur, ${ }^{55}$ P. K. Behera ${ }^{56}$ R. Chudasama,${ }^{57}$ D. Dutta, ${ }^{57}$ V. Jha, ${ }^{57}$ V. Kumar,${ }^{57}$ A. K. Mohanty, ${ }^{57, \mathrm{r}}$ P. K. Netrakanti, ${ }^{57}$ L. M. Pant ${ }^{57}$ P. Shukla, ${ }^{57}$ A. Topkar, ${ }^{57}$ T. Aziz, ${ }^{58}$ S. Dugad ${ }^{58}$ B. Mahakud ${ }^{58}$ S. Mitra, ${ }^{58}$ G. B. Mohanty, ${ }^{58}$ N. Sur,${ }^{58}$ B. Sutar, ${ }^{58}$ S. Banerjee, ${ }^{59}$ S. Bhattacharya, ${ }^{59}$ S. Chatterjee, ${ }^{59}$ P. Das, ${ }^{59}$ M. Guchait,${ }^{59}$ Sa. Jain, ${ }^{59}$ S. Kumar,${ }^{59}$ M. Maity, ${ }^{59}$,aa G. Majumder, ${ }^{59}$ K. Mazumdar, ${ }^{59}$ T. Sarkar, ${ }^{59, \text { aa }}$ N. Wickramage, ${ }^{59, \text { bb }}$ S. Chauhan ${ }^{60}$ S. Dube, ${ }^{60}$ V. Hegde,${ }^{60}$ A. Kapoor, ${ }^{60}$ K. Kothekar, ${ }^{60}$ S. Pandey, ${ }^{60}$ A. Rane,${ }^{60}$ S. Sharma, ${ }^{60}$ S. Chenarani, ${ }^{61, c c}$ E. Eskandari Tadavani,${ }^{61}$ S. M. Etesami, ${ }^{61, c c}$ M. Khakzad, ${ }^{61}$ M. Mohammadi Najafabadi, ${ }^{61}$ M. Naseri, ${ }^{61}$ S. Paktinat Mehdiabadi, ${ }^{61, d d}$ F. Rezaei Hosseinabadi, ${ }^{61}$ B. Safarzadeh, ${ }^{61, e e}$ M. Zeinali, ${ }^{61}$ M. Felcini, ${ }^{62}$ M. Grunewald ${ }^{62}$ M. Abbrescia, ${ }^{6 a, 63 b}$ C. Calabria, ${ }^{63 a, 63 b}$ A. Colaleo, ${ }^{63 a}$ D. Creanza, ${ }^{63 a, 63 c}$ L. Cristella, ${ }^{63 a, 63 b}$ N. De Filippis, ${ }^{63 a, 63 c}$ M. De Palma, ${ }^{63 a, 63 b}$ F. Errico, ${ }^{63 a, 63 b}$ L. Fiore, ${ }^{63 a}$ G. Iaselli, ${ }^{63 a, 63 c}$ S. Lezki, ${ }^{63 a, 63 b}$ G. Maggi, ${ }^{63 a, 63 c}$ M. Maggi, ${ }^{63 a}$ G. Miniello, ${ }^{63 a, 63 b}$ S. My, ${ }^{63 a, 63 b}$ S. Nuzzo, ${ }^{63 a, 63 b}$ A. Pompili, ${ }^{63 a, 63 b}$ G. Pugliese, ${ }^{63 a, 63 c}$ R. Radogna, ${ }^{63 a}$ A. Ranieri, ${ }^{63 a}$ G. Selvaggi ${ }^{63 a, 63 b}$ A. Sharma, ${ }^{63 a}$ L. Silvestris, ${ }^{63 a, r}$ R. Venditti, ${ }^{63 a}$ P. Verwilligen, ${ }^{63 \mathrm{a}}$ G. Abbiendi, ${ }^{64 \mathrm{a}}$ C. Battilana, ${ }^{64 \mathrm{a}, 64 \mathrm{~b}}$ D. Bonacorsi, ${ }^{64 \mathrm{a}, 64 \mathrm{~b}}$ L. Borgonovi, ${ }^{64 \mathrm{a}, 64 \mathrm{~b}} \mathrm{~S}$. Braibant-Giacomelli, ${ }^{64 \mathrm{a}, 64 \mathrm{~b}}$ R. Campanini, ${ }^{64 a, 64 b}$ P. Capiluppi, ${ }^{64 a, 64 b}$ A. Castro, ${ }^{64 a, 64 b}$ F. R. Cavallo, ${ }^{64 a}$ S. S. Chhibra, ${ }^{64 a}$ G. Codispoti, ${ }^{64 a, 64 b}$ M. Cuffiani, ${ }^{64 a, 64 b}$ G. M. Dallavalle, ${ }^{64 a}$ F. Fabbri, ${ }^{64 a}$ A. Fanfani, ${ }^{64 a, 64 b}$ D. Fasanella, ${ }^{64 a, 64 b}$ P. Giacomelli, ${ }^{64 a}$ C. Grandi, ${ }^{64 a}$ L. Guiducci, ${ }^{64 a, 64 b}$ S. Marcellini, ${ }^{64 a}$ G. Masetti, ${ }^{64 a}$ A. Montanari, ${ }^{64 a}$ F. L. Navarria,${ }^{64 a, 64 b}$ A. Perrotta, ${ }^{64 a}$ A. M. Rossi ${ }^{64 a, 64 b}$ T. Rovelli, ${ }^{64 a, 64 b}$ G. P. Siroli, ${ }^{64 a, 64 b}$ N. Tosi ${ }^{64 a}$ S. Albergo, ${ }^{65 a, 65 b}$ S. Costa ${ }^{65 a, 65 b}$ A. Di Mattia, ${ }^{65 a}$ F. Giordano, ${ }^{65 a, 65 b}$ R. Potenza, ${ }^{65 a, 65 b}$ A. Tricomi, ${ }^{65 a, 65 b}$ C. Tuve, ${ }^{65 a, 65 b}$ G. Barbagli, ${ }^{66 a}$ K. Chatterjee, ${ }^{66 a, 66 b}$ V. Ciulli, ${ }^{66 a, 66 b}$ C. Civinini, ${ }^{66 a}$ R. D’Alessandro, ${ }^{66 a, 66 b}$ E. Focardi, ${ }^{66 a, 66 b}$ P. Lenzi, ${ }^{66 a, 66 b}$ M. Meschini, ${ }^{66 a}$ S. Paoletti ${ }^{66 \mathrm{a}}$ L. Russo, ${ }^{66 a, f f}$ G. Sguazzoni, ${ }^{66 a}$ D. Strom, ${ }^{66 \mathrm{a}}$ L. Viliani, ${ }^{66 \mathrm{a}, 66 \mathrm{~b}, \mathrm{r}}$ L. Benussi, ${ }^{67}$ S. Bianco, ${ }^{67}$ F. Fabbri, ${ }^{67}$ D. Piccolo,${ }^{67}$ F. Primavera, ${ }^{67, \mathrm{r}}$ V. Calvelli, ${ }^{68 \mathrm{a}, 68 \mathrm{~b}}$ F. Ferro, ${ }^{68 a}$ E. Robutti, ${ }^{68 a}$ S. Tosi, ${ }^{68 a, 68 b}$ A. Benaglia, ${ }^{69 a}$ A. Beschi, ${ }^{69 a}$ L. Brianza,${ }^{69 a, 69 b}$ F. Brivio, ${ }^{69 a, 69 b}$ V. Ciriolo, ${ }^{69 a, 69 b}$ M. E. Dinardo, ${ }^{69 a, 69 b}$ S. Fiorendi, ${ }^{69 a, 69 b}$ S. Gennai, ${ }^{69 a}$ A. Ghezzi, ${ }^{69 a, 69 b}$ P. Govoni, ${ }^{69,69 b}$ M. Malberti, ${ }^{69 a, 69 b}$ S. Malvezzi, ${ }^{69 a}$ R. A. Manzoni,${ }^{69 a, 69 b}$ D. Menasce, ${ }^{69 a}$ L. Moroni, ${ }^{69 a}$ M. Paganoni, ${ }^{69 a, 69 b}$ K. Pauwels ${ }^{69 a, 69 b}$ D. Pedrini, ${ }^{69 a}$ S. Pigazzini,${ }^{69,69 b, g g}$ S. Ragazzi ${ }^{69 a, 69 b}$ N. Redaelli, ${ }^{69 a}$ T. Tabarelli de Fatis,${ }^{69 a, 69 b}$ S. Buontempo,${ }^{70 a}$ N. Cavallo, ${ }^{70 a, 70 c}$ S. Di Guida, ${ }^{70 a, 70 d, r}$ F. Fabozzi, ${ }^{70 a, 70 c}$ F. Fienga, ${ }^{70 a, 70 b}$ A. O. M. Iorio, ${ }^{70 a, 70 b}$ W. A. Khan, ${ }^{70 a}$ L. Lista, ${ }^{70 a}$ S. Meola ${ }^{70 a, 70 d, r}$ P. Paolucci, ${ }^{70 a, r}$ C. Sciacca, ${ }^{70 a, 70 b}$ F. Thyssen, ${ }^{70 a}$ P. Azzi, ${ }^{71 a}$ N. Bacchetta, ${ }^{71 a}$ L. Benato, ${ }^{71 a, 71 b}$ D. Bisello, ${ }^{71 a, 71 b}$ A. Boletti, ${ }^{71 a, 71 b}$ R. Carlin, ${ }^{71,71 b}$ A. Carvalho Antunes De Oliveira, ${ }^{71 a, 71 b}$ P. Checchia, ${ }^{71 a}$ P. De Castro Manzano, ${ }^{71 a}$ T. Dorigo, ${ }^{71 a}$ U. Dosselli, ${ }^{71 \mathrm{a}}$ F. Gasparini, ${ }^{71,71 \mathrm{~b}}$ U. Gasparini, ${ }^{71 \mathrm{a}, 71 \mathrm{~b}}$ A. Gozzelino, ${ }^{71 \mathrm{a}}$ S. Lacaprara, ${ }^{71 \mathrm{a}}$ M. Margoni, ${ }^{7 \mathrm{a}, 7 \mathrm{~b}}$ A. T. Meneguzzo, ${ }^{71 \mathrm{a}, 71 \mathrm{~b}}$ N. Pozzobon, ${ }^{71 \mathrm{a}, 71 \mathrm{~b}}$ P. Ronchese, ${ }^{71 \mathrm{a}, 71 \mathrm{~b}}$ R. Rossin, ${ }^{71 \mathrm{a}, 71 \mathrm{~b}}$ F. Simonetto, ${ }^{71 \mathrm{a}, 71 \mathrm{~b}}$ E. Torassa, ${ }^{71 \mathrm{a}}$ M. Zanetti, ${ }^{71 a, 71 b}$ P. Zotto, ${ }^{71 a, 71 b}$ G. Zumerle, ${ }^{71 a, 71 b}$ A. Braghieri, ${ }^{72 a}$ A. Magnani, ${ }^{72 a}$ P. Montagna, ${ }^{72 a, 72 b}$ S. P. Ratti, ${ }^{72 a, 72 b}$ V. Re, ${ }^{72 a}$ M. Ressegotti, ${ }^{72 a, 72 b}$ C. Riccardi, ${ }^{72 a, 72 b}$ P. Salvini, ${ }^{72 a}$ I. Vai, ${ }^{72 a, 72 b}$ P. Vitulo, ${ }^{72 a, 72 b}$ L. Alunni Solestizi, ${ }^{73 a, 73 b}$ M. Biasini, ${ }^{73 a, 73 b}$ G. M. Bilei, ${ }^{73 a}$ C. Cecchi, ${ }^{73 a, 73 b}$ D. Ciangottini, ${ }^{73 a, 73 b}$ L. Fanò, ${ }^{73 a, 73 b}$ P. Lariccia, ${ }^{73 a, 73 b}$ R. Leonardi, ${ }^{73 a, 73 b}$ E. Manoni, ${ }^{73 a}$ G. Mantovani, ${ }^{73 a, 73 b}$ V. Mariani, ${ }^{73 a, 73 b}$ M. Menichelli, ${ }^{73 a}$ A. Rossi, ${ }^{73 a, 73 b}$ A. Santocchia, ${ }^{73 a, 73 b}$ D. Spiga, ${ }^{73 a}$ K. Androsov, ${ }^{74 a}$ P. Azzurri, ${ }^{74 a, r}$ G. Bagliesi, ${ }^{74 a}$ T. Boccali, ${ }^{74 a}$ L. Borrello, ${ }^{74 a}$ R. Castaldi, ${ }^{74 a}$ M. A. Ciocci, ${ }^{74 a, 74 b}$

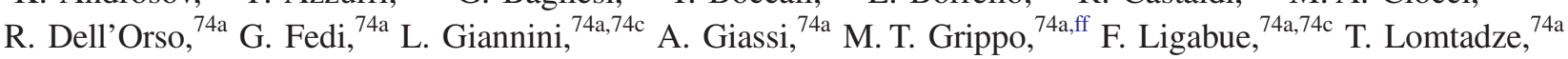
E. Manca, ${ }^{74 a, 74 c}$ G. Mandorli, ${ }^{74 a, 74 c}$ L. Martini, ${ }^{74 a, 74 b}$ A. Messineo, ${ }^{74 a, 74 b}$ F. Palla, ${ }^{74 a}$ A. Rizzi, ${ }^{74 a, 74 b}$ A. Savoy-Navarro, ${ }^{74 a, \text {,h }}$ P. Spagnolo ${ }^{74 a}$ R. Tenchini, ${ }^{74 a}$ G. Tonelli, ${ }^{74 a}, 74 b$ A. Venturi, ${ }^{74 a}$ P. G. Verdini, ${ }^{74 a}$ L. Barone,${ }^{75 a, 75 b}$ F. Cavallari, ${ }^{75 a}$ M. Cipriani ${ }^{75 a, 75 b}$ N. Daci, ${ }^{75 a}$ D. Del Re,${ }^{75 a, 75 b, r}$ E. Di Marco, ${ }^{75 a, 75 b}$ M. Diemoz, ${ }^{75 a}$ S. Gelli, ${ }^{75 a, 75 b}$ E. Longo, ${ }^{75 a, 75 b}$ F. Margaroli, ${ }^{75 a, 75 b}$ B. Marzocchi, ${ }^{75 a, 75 b}$ P. Meridiani, ${ }^{75 a}$ G. Organtini, ${ }^{75 a, 75 b}$ R. Paramatti, ${ }^{75 a, 75 b}$ F. Preiato,${ }^{75 a, 75 b}$ S. Rahatlou, ${ }^{75 a, 75 b}$ C. Rovelli, ${ }^{75 a}$ F. Santanastasio, ${ }^{75 a, 75 b}$ N. Amapane, ${ }^{76 a, 76 b}$ R. Arcidiacono, ${ }^{76 a, 76 c}$ S. Argiro, ${ }^{76 a, 76 b}$ M. Arneodo, ${ }^{76 a, 76 c}$ N. Bartosik, ${ }^{76 a}$ R. Bellan, ${ }^{76 a, 76 b}$ C. Biino, ${ }^{76 a}$ N. Cartiglia,${ }^{76 a}$ F. Cenna, ${ }^{76 a, 76 b}$ M. Costa, ${ }^{76 a, 76 b}$ R. Covarelli, ${ }^{76 a, 76 b}$ A. Degano, ${ }^{76 a, 76 b}$ N. Demaria, ${ }^{76 a}$ B. Kiani, ${ }^{76 a, 76 b}$ C. Mariotti, ${ }^{76 a}$ S. Maselli, ${ }^{76 a}$ E. Migliore, ${ }^{76 a, 76 b}$ V. Monaco, ${ }^{76 a, 76 b}$ E. Monteil, ${ }^{76 a, 76 b}$ M. Monteno, ${ }^{76 a}$ M. M. Obertino, ${ }^{76 a, 76 b}$ L. Pacher, ${ }^{76 a, 76 b}$ N. Pastrone, ${ }^{76 a}$ M. Pelliccioni, ${ }^{76 a}$ 
G. L. Pinna Angioni, ${ }^{76 a, 76 b}$ F. Ravera, ${ }^{76 a, 76 b}$ A. Romero, ${ }^{76 a, 76 b}$ M. Ruspa,${ }^{76 a, 76 c}$ R. Sacchi, ${ }^{76 a, 76 b}$ K. Shchelina,${ }^{76 a, 76 b}$ V. Sola, ${ }^{76 a}$ A. Solano, ${ }^{76 a, 76 b}$ A. Staiano, ${ }^{76 a}$ P. Traczyk, ${ }^{76 a, 76 b}$ S. Belforte, ${ }^{77 a}$ M. Casarsa, ${ }^{77 a}$ F. Cossutti, ${ }^{77 a}$ G. Della Ricca, ${ }^{77 a, 77 b}$ A. Zanetti, ${ }^{77 a}$ D. H. Kim, ${ }^{78}$ G. N. Kim, ${ }^{78}$ M. S. Kim, ${ }^{78}$ J. Lee, ${ }^{78}$ S. Lee, ${ }^{78}$ S. W. Lee, ${ }^{78}$ C. S. Moon,${ }^{78}$ Y. D. Oh, ${ }^{78}$ S. Sekmen, ${ }^{78}$ D. C. Son, ${ }^{78}$ Y. C. Yang, ${ }^{78}$ A. Lee, ${ }^{79}$ H. Kim, ${ }^{80}$ D. H. Moon,${ }^{80}$ G. Oh ${ }^{80}$ J. A. Brochero Cifuentes, ${ }^{81}$ J. Goh, ${ }^{81}$ T. J. Kim, ${ }^{81}$ S. Cho, ${ }^{82}$ S. Choi ${ }^{82}$ Y. Go, ${ }^{82}$ D. Gyun, ${ }^{82}$ S. Ha, ${ }^{82}$ B. Hong, ${ }^{82}$ Y. Jo, ${ }^{82}$ Y. Kim,${ }^{82}$ K. Lee, ${ }^{82}$ K. S. Lee, ${ }^{82}$ S. Lee, ${ }^{82}$ J. Lim, ${ }^{82}$ S. K. Park, ${ }^{82}$ Y. Roh, ${ }^{82}$ J. Almond, ${ }^{83}$ J. Kim, ${ }^{83}$ J. S. Kim, ${ }^{83}$ H. Lee, ${ }^{83}$ K. Lee, ${ }^{83}$ K. Nam, ${ }^{83}$ S. B. Oh, ${ }^{83}$ B. C. Radburn-Smith, ${ }^{83}$ S. h. Seo, ${ }^{83}$ U. K. Yang, ${ }^{83}$ H. D. Yoo, ${ }^{83}$ G. B. Yu,${ }^{83}$ M. Choi, ${ }^{84}$ H. Kim, ${ }^{84}$ J. H. Kim, ${ }^{84}$ J. S. H. Lee, ${ }^{84}$ I. C. Park, ${ }^{84}$ Y. Choi ${ }^{85}$ C. Hwang, ${ }^{85}$ J. Lee, ${ }^{85}$ I. Yu, ${ }^{85}$ V. Dudenas, ${ }^{86}$ A. Juodagalvis, ${ }^{86}$ J. Vaitkus, ${ }^{86}$ I. Ahmed,${ }^{87}$ Z. A. Ibrahim, ${ }^{87}$ M. A. B. Md Ali, ${ }^{87, i i}$ F. Mohamad Idris, ${ }^{87, j j}$ W. A. T. Wan Abdullah, ${ }^{87}$ M. N. Yusli, ${ }^{87}$ Z. Zolkapli, ${ }^{87}$ R Reyes-Almanza, ${ }^{88}$ G. Ramirez-Sanchez ${ }^{88}$ M. C. Duran-Osuna, ${ }^{88}$ H. Castilla-Valdez ${ }^{88}$ E. De La Cruz-Burelo, ${ }^{88}$ I. Heredia-De La Cruz, ${ }^{88, k k}$ R. I. Rabadan-Trejo, ${ }^{88}$ R. Lopez-Fernandez, ${ }^{88}$ J. Mejia Guisao, ${ }^{88}$ A. Sanchez-Hernandez ${ }^{88}$ S. Carrillo Moreno, ${ }^{89}$ C. Oropeza Barrera, ${ }^{89}$ F. Vazquez Valencia,${ }^{89}$ I. Pedraza, ${ }^{90}$ H. A. Salazar Ibarguen, ${ }^{90}$ C. Uribe Estrada,${ }^{90}$ A. Morelos Pineda, ${ }^{91}$ D. Krofcheck,${ }^{92}$ P. H. Butler, ${ }^{93}$ A. Ahmad, ${ }^{94}$ M. Ahmad,${ }^{94}$ Q. Hassan, ${ }^{94}$ H. R. Hoorani, ${ }^{94}$ A. Saddique,${ }^{94}$ M. A. Shah,${ }^{94}$ M. Shoaib, ${ }^{94}$ M. Waqas,${ }^{94}$ H. Bialkowska, ${ }^{95}$ M. Bluj, ${ }^{95}$ B. Boimska, ${ }^{95}$ T. Frueboes,${ }^{95}$ M. Górski,${ }^{95}$ M. Kazana, ${ }^{95}$ K. Nawrocki, ${ }^{95}$ M. Szleper, ${ }^{95}$ P. Zalewski, ${ }^{95}$ K. Bunkowski, ${ }^{96}$ A. Byszuk, ${ }^{96,11}$ K. Doroba,${ }^{96}$ A. Kalinowski, ${ }^{96}$ M. Konecki, ${ }^{96}$ J. Krolikowski, ${ }^{96}$ M. Misiura, ${ }^{96}$ M. Olszewski, ${ }^{96}$ A. Pyskir,${ }^{96}$ M. Walczak, ${ }^{96}$ P. Bargassa, ${ }^{97}$ C. Beirão Da Cruz E Silva, ${ }^{97}$ A. Di Francesco, ${ }^{97}$ P. Faccioli, ${ }^{97}$ B. Galinhas, ${ }^{97}$ M. Gallinaro, ${ }^{97}$ J. Hollar, ${ }^{97}$ N. Leonardo, ${ }^{97}$ L. Lloret Iglesias, ${ }^{97}$ M. V. Nemallapudi, ${ }^{97}$ J. Seixas,${ }^{97}$

G. Strong, ${ }^{97}$ O. Toldaiev, ${ }^{97}$ D. Vadruccio, ${ }^{97}$ J. Varela,${ }^{97}$ S. Afanasiev,${ }^{98}$ P. Bunin,${ }^{98}$ M. Gavrilenko, ${ }^{98}$ I. Golutvin,${ }^{98}$ I. Gorbunov, ${ }^{98}$ A. Kamenev, ${ }^{98}$ V. Karjavin, ${ }^{98}$ A. Lanev, ${ }^{98}$ A. Malakhov, ${ }^{98}$ V. Matveev, ${ }^{98, m m, n n}$ V. Palichik,${ }^{98}$ V. Perelygin,${ }^{98}$ S. Shmatov, ${ }^{98}$ S. Shulha, ${ }^{98}$ N. Skatchkov, ${ }^{98}$ V. Smirnov, ${ }^{98}$ N. Voytishin, ${ }^{98}$ A. Zarubin, ${ }^{98}$ Y. Ivanov, ${ }^{99}$ V. Kim, ${ }^{99,00}$ E. Kuznetsova, ${ }^{99, p p}$ P. Levchenko, ${ }^{99}$ V. Murzin, ${ }^{99}$ V. Oreshkin, ${ }^{99}$ I. Smirnov, ${ }^{99}$ V. Sulimov, ${ }^{99}$ L. Uvarov, ${ }^{99}$ S. Vavilov, ${ }^{99}$ A. Vorobyev, ${ }^{99}$ Yu. Andreev, ${ }^{100}$ A. Dermenev, ${ }^{100}$ S. Gninenko, ${ }^{100}$ N. Golubev, ${ }^{100}$ A. Karneyeu, ${ }^{100}$ M. Kirsanov, ${ }^{100}$ N. Krasnikov, ${ }^{100}$ A. Pashenkov, ${ }^{100}$ D. Tlisov, ${ }^{100}$ A. Toropin, ${ }^{100}$ V. Epshteyn,${ }^{101}$ V. Gavrilov, ${ }^{101}$ N. Lychkovskaya,${ }^{101}$ V. Popov, ${ }^{101}$ I. Pozdnyakov, ${ }^{101}$ G. Safronov, ${ }^{101}$ A. Spiridonov, ${ }^{101}$ A. Stepennov, ${ }^{101}$ M. Toms,${ }^{101}$ E. Vlasov, ${ }^{101}$ A. Zhokin, ${ }^{101}$ T. Aushev, ${ }^{102}$ A. Bylinkin, ${ }^{102, \mathrm{nn}}$ M. Chadeeva, ${ }^{103, q \mathrm{q}}$ O. Markin, ${ }^{103}$ P. Parygin, ${ }^{103}$ D. Philippov, ${ }^{103}$ S. Polikarpov, ${ }^{103}$ V. Rusinov, ${ }^{103}$ E. Zhemchugov, ${ }^{103}$ V. Andreev, ${ }^{104}$ M. Azarkin, ${ }^{104, n n}$ I. Dremin, ${ }^{104, n n}$ M. Kirakosyan, ${ }^{104, n n}$ A. Terkulov, ${ }^{104}$ A. Baskakov, ${ }^{105}$ A. Belyaev, ${ }^{105}$ E. Boos, ${ }^{105}$ V. Bunichev, ${ }^{105}$ M. Dubinin, ${ }^{105, r \mathrm{rr}}$ L. Dudko, ${ }^{105}$ A. Gribushin, ${ }^{105}$ V. Klyukhin, ${ }^{105}$ O. Kodolova, ${ }^{105}$ I. Lokhtin, ${ }^{105}$ I. Miagkov, ${ }^{105}$ S. Obraztsov, ${ }^{105}$ S. Petrushanko, ${ }^{105}$ V. Savrin, ${ }^{105}$ A. Snigirev,${ }^{105}$ V. Blinov,${ }^{106, s s}$ Y. Skovpen, ${ }^{106, s s}$ D. Shtol,${ }^{106, s s}$ I. Azhgirey, ${ }^{107}$ I. Bayshev, ${ }^{107}$ S. Bitioukov, ${ }^{107}$ D. Elumakhov, ${ }^{107}$ V. Kachanov, ${ }^{107}$ A. Kalinin, ${ }^{107}$ D. Konstantinov, ${ }^{107}$ P. Mandrik, ${ }^{107}$ V. Petrov, ${ }^{107}$ R. Ryutin, ${ }^{107}$ A. Sobol, ${ }^{107}$ S. Troshin,${ }^{107}$ N. Tyurin, ${ }^{107}$ A. Uzunian, ${ }^{107}$ A. Volkov, ${ }^{107}$ P. Adzic, ${ }^{108, t t}$ P. Cirkovic, ${ }^{108}$ D. Devetak, ${ }^{108}$ M. Dordevic, ${ }^{108}$ J. Milosevic, ${ }^{108}$ V. Rekovic, ${ }^{108}$ J. Alcaraz Maestre, ${ }^{109}$ M. Barrio Luna, ${ }^{109}$ M. Cerrada,${ }^{109}$ N. Colino, ${ }^{109}$ B. De La Cruz, ${ }^{109}$ A. Delgado Peris, ${ }^{109}$

A. Escalante Del Valle, ${ }^{109}$ C. Fernandez Bedoya,${ }^{109}$ J. P. Fernández Ramos, ${ }^{109}$ J. Flix, ${ }^{109}$ M. C. Fouz, ${ }^{109}$

O. Gonzalez Lopez, ${ }^{109}$ S. Goy Lopez, ${ }^{109}$ J. M. Hernandez, ${ }^{109}$ M. I. Josa, ${ }^{109}$ D. Moran, ${ }^{109}$ A. Pérez-Calero Yzquierdo, ${ }^{109}$ J. Puerta Pelayo, ${ }^{109}$ A. Quintario Olmeda, ${ }^{109}$ I. Redondo, ${ }^{109}$ L. Romero, ${ }^{109}$ M. S. Soares, ${ }^{109}$ A. Álvarez Fernández, ${ }^{109}$ C. Albajar, ${ }^{110}$ J. F. de Trocóniz, ${ }^{110}$ M. Missiroli, ${ }^{110}$ J. Cuevas, ${ }^{111}$ C. Erice, ${ }^{111}$ J. Fernandez Menendez, ${ }^{111}$

I. Gonzalez Caballero, ${ }^{111}$ J. R. González Fernández, ${ }^{111}$ E. Palencia Cortezon, ${ }^{111}$ S. Sanchez Cruz, ${ }^{111}$ P. Vischia, ${ }^{111}$ J. M. Vizan Garcia, ${ }^{111}$ I. J. Cabrillo, ${ }^{112}$ A. Calderon, ${ }^{112}$ B. Chazin Quero, ${ }^{112}$ E. Curras, ${ }^{112}$ J. Duarte Campderros, ${ }^{112}$ M. Fernandez, ${ }^{112}$ J. Garcia-Ferrero, ${ }^{112}$ G. Gomez, ${ }^{112}$ A. Lopez Virto, ${ }^{112}$ J. Marco, ${ }^{112}$ C. Martinez Rivero, ${ }^{112}$ P. Martinez Ruiz del Arbol, ${ }^{112}$ F. Matorras, ${ }^{112}$ J. Piedra Gomez, ${ }^{112}$ T. Rodrigo, ${ }^{112}$ A. Ruiz-Jimeno, ${ }^{112}$ L. Scodellaro, ${ }^{112}$ N. Trevisani, ${ }^{112}$ I. Vila, ${ }^{112}$ R. Vilar Cortabitarte, ${ }^{112}$ D. Abbaneo, ${ }^{113}$ B. Akgun, ${ }^{113}$ E. Auffray, ${ }^{113}$ P. Baillon, ${ }^{113}$ A. H. Ball, ${ }^{113}$ D. Barney, ${ }^{113}$ J. Bendavid, ${ }^{113}$ M. Bianco, ${ }^{113}$ P. Bloch, ${ }^{113}$ A. Bocci, ${ }^{113}$ C. Botta, ${ }^{113}$ T. Camporesi, ${ }^{113}$ R. Castello, ${ }^{113}$ M. Cepeda, ${ }^{113}$ G. Cerminara, ${ }^{113}$ E. Chapon, ${ }^{113}$ Y. Chen, ${ }^{113}$ D. d'Enterria, ${ }^{113}$ A. Dabrowski, ${ }^{113}$ V. Daponte, ${ }^{113}$ A. David, ${ }^{113}$ M. De Gruttola, ${ }^{113}$ A. De Roeck, ${ }^{113}$ N. Deelen, ${ }^{113}$ M. Dobson, ${ }^{113}$ T. du Pree, ${ }^{113}$ M. Dünser, ${ }^{113}$ N. Dupont, ${ }^{113}$ A. Elliott-Peisert, ${ }^{113}$ P. Everaerts, ${ }^{113}$ F. Fallavollita, ${ }^{113}$ G. Franzoni, ${ }^{113}$ J. Fulcher, ${ }^{113}$ W. Funk, ${ }^{113}$ D. Gigi, ${ }^{113}$ A. Gilbert, ${ }^{113}$ K. Gill, ${ }^{113}$ F. Glege, ${ }^{113}$ D. Gulhan, ${ }^{113}$ P. Harris, ${ }^{113}$ J. Hegeman, ${ }^{113}$ V. Innocente, ${ }^{113}$ A. Jafari, ${ }^{113}$ P. Janot, ${ }^{113}$ O. Karacheban, ${ }^{113, u}$ J. Kieseler, ${ }^{113}$ V. Knünz, ${ }^{113}$ A. Kornmayer, ${ }^{113}$ M. J. Kortelainen, ${ }^{113}$ M. Krammer, ${ }^{113, b}$ C. Lange, ${ }^{113}$ P. Lecoq, ${ }^{113}$ C. Lourenço, ${ }^{113}$ M. T. Lucchini, ${ }^{113}$ L. Malgeri, ${ }^{113}$ M. Mannelli, ${ }^{113}$ A. Martelli, ${ }^{113}$ F. Meijers, ${ }^{113}$ J. A. Merlin, ${ }^{113}$ S. Mersi, ${ }^{113}$ E. Meschi, ${ }^{113}$ P. Milenovic, ${ }^{113, \text { uu }}$ F. Moortgat, ${ }^{113}$ M. Mulders, ${ }^{113}$ H. Neugebauer, ${ }^{113}$ J. Ngadiuba, ${ }^{113}$ 
S. Orfanelli, ${ }^{113}$ L. Orsini, ${ }^{113}$ L. Pape, ${ }^{113}$ E. Perez, ${ }^{113}$ M. Peruzzi, ${ }^{113}$ A. Petrilli, ${ }^{113}$ G. Petrucciani, ${ }^{113}$ A. Pfeiffer,${ }^{113}$ M. Pierini, ${ }^{113}$ D. Rabady, ${ }^{113}$ A. Racz, ${ }^{113}$ T. Reis, ${ }^{113}$ G. Rolandi, ${ }^{113, v v}$ M. Rovere,${ }^{113}$ H. Sakulin, ${ }^{113}$ C. Schäfer, ${ }^{113}$ C. Schwick, ${ }^{113}$ M. Seidel, ${ }^{113}$ M. Selvaggi, ${ }^{113}$ A. Sharma, ${ }^{113}$ P. Silva, ${ }^{113}$ P. Sphicas, ${ }^{113, \text { ww }}$ A. Stakia, ${ }^{113}$ J. Steggemann, ${ }^{113}$ M. Stoye ${ }^{113}$ M. Tosi, ${ }^{113}$ D. Treille, ${ }^{113}$ A. Triossi, ${ }^{113}$ A. Tsirou, ${ }^{113}$ V. Veckalns, ${ }^{113, x x}$ M. Verweij,${ }^{113}$ W. D. Zeuner,${ }^{113}$ W. Bertl, ${ }^{114, a}$ L. Caminada, ${ }^{114, y y}$ K. Deiters, ${ }^{114}$ W. Erdmann, ${ }^{114}$ R. Horisberger, ${ }^{114}$ Q. Ingram, ${ }^{114}$ H. C. Kaestli, ${ }^{114}$ D. Kotlinski, ${ }^{114}$ U. Langenegger, ${ }^{114}$ T. Rohe, ${ }^{114}$ S. A. Wiederkehr, ${ }^{114}$ M. Backhaus,${ }^{115}$ L. Bäni, ${ }^{115}$ P. Berger,${ }^{115}$ L. Bianchini, ${ }^{115}$ B. Casal, ${ }^{115}$ G. Dissertori, ${ }^{115}$ M. Dittmar, ${ }^{115}$ M. Donegà, ${ }^{115}$ C. Dorfer, ${ }^{115}$ C. Grab, ${ }^{115}$ C. Heidegger, ${ }^{115}$

D. Hits, ${ }^{115}$ J. Hoss,${ }^{115}$ G. Kasieczka, ${ }^{115}$ T. Klijnsma, ${ }^{115}$ W. Lustermann, ${ }^{115}$ B. Mangano, ${ }^{115}$ M. Marionneau, ${ }^{115}$ M. T. Meinhard ${ }^{115}$ D. Meister, ${ }^{115}$ F. Micheli, ${ }^{115}$ P. Musella, ${ }^{115}$ F. Nessi-Tedaldi, ${ }^{115}$ F. Pandolfi, ${ }^{115}$ J. Pata, ${ }^{115}$ F. Pauss, ${ }^{115}$ G. Perrin, ${ }^{115}$ L. Perrozzi, ${ }^{115}$ M. Quittnat, ${ }^{115}$ M. Reichmann, ${ }^{115}$ D. A. Sanz Becerra,${ }^{115}$ M. Schönenberger, ${ }^{115}$ L. Shchutska, ${ }^{115}$ V. R. Tavolaro, ${ }^{115}$ K. Theofilatos, ${ }^{115}$ M. L. Vesterbacka Olsson, ${ }^{115}$ R. Wallny, ${ }^{115}$ D. H. Zhu, ${ }^{115}$ T. K. Aarrestad, ${ }^{116}$ C. Amsler, ${ }^{116, z z}$ M. F. Canelli, ${ }^{116}$ A. De Cosa, ${ }^{116}$ R. Del Burgo,${ }^{116}$ S. Donato, ${ }^{116}$ C. Galloni, ${ }^{116}$ T. Hreus,${ }^{116}$ B. Kilminster, ${ }^{116}$ D. Pinna, ${ }^{116}$ G. Rauco, ${ }^{116}$ P. Robmann, ${ }^{116}$ D. Salerno, ${ }^{116}$ K. Schweiger, ${ }^{116}$ C. Seitz, ${ }^{116}$ Y. Takahashi, ${ }^{116}$ A. Zucchetta, ${ }^{116}$ V. Candelise, ${ }^{117}$ T. H. Doan, ${ }^{117}$ Sh. Jain, ${ }^{117}$ R. Khurana, ${ }^{117}$ C. M. Kuo, ${ }^{117}$ W. Lin, ${ }^{117}$ A. Pozdnyakov, ${ }^{117}$ S. S. Yu, ${ }^{117}$ Arun Kumar, ${ }^{118}$ P. Chang, ${ }^{118}$ Y. Chao, ${ }^{118}$ K. F. Chen, ${ }^{118}$ P. H. Chen, ${ }^{118}$ F. Fiori, ${ }^{118}$ W.-S. Hou, ${ }^{118}$ Y. Hsiung, ${ }^{118}$ Y. F. Liu, ${ }^{118}$ R.-S. Lu, ${ }^{118}$ E. Paganis, ${ }^{118}$ A. Psallidas, ${ }^{118}$ A. Steen, ${ }^{118}$ J. f. Tsai, ${ }^{118}$ B. Asavapibhop, ${ }^{119}$ K. Kovitanggoon, ${ }^{119}$ G. Singh, ${ }^{119}$

N. Srimanobhas, ${ }^{119}$ A. Bat, ${ }^{120}$ F. Boran, ${ }^{120}$ S. Cerci, ${ }^{120, \text { aaa }}$ S. Damarseckin, ${ }^{120}$ Z. S. Demiroglu, ${ }^{120}$ C. Dozen, ${ }^{120}$ I. Dumanoglu, ${ }^{120}$ S. Girgis, ${ }^{120}$ G. Gokbulut, ${ }^{120}$ Y. Guler, ${ }^{120}$ I. Hos, ${ }^{120, b b b}$ E. E. Kangal, ${ }^{120, c c c}$ O. Kara, ${ }^{120}$ A. Kayis Topaksu, ${ }^{120}$ U. Kiminsu, ${ }^{120}$ M. Oglakci, ${ }^{120}$ G. Onengut, ${ }^{120, d d d}$ K. Ozdemir, ${ }^{120, \text { eee }}$ D. Sunar Cerci, ${ }^{120, \text { aaa }}$ B. Tali, ${ }^{120 \text {,aa }}$ U. G. Tok, ${ }^{120}$ S. Turkcapar, ${ }^{120}$ I. S. Zorbakir, ${ }^{120}$ C. Zorbilmez, ${ }^{120}$ B. Bilin, ${ }^{121}$ G. Karapinar, ${ }^{121, f f f}$ K. Ocalan, ${ }^{121, \text { ggg }}$ M. Yalvac, ${ }^{121}$ M. Zeyrek, ${ }^{121}$ E. Gülmez, ${ }^{122}$ M. Kaya, ${ }^{122, \text { hhh }}$ O. Kaya, ${ }^{122, \text { iii }}$ S. Tekten, ${ }^{122}$ E. A. Yetkin, ${ }^{122, j j j}$ M. N. Agaras, ${ }^{123}$ S. Atay, ${ }^{123}$ A. Cakir, ${ }^{123}$ K. Cankocak, ${ }^{123}$ B. Grynyov, ${ }^{124}$ L. Levchuk, ${ }^{125}$ F. Ball, ${ }^{126}$ L. Beck, ${ }^{126}$ J. J. Brooke, ${ }^{126}$ D. Burns, ${ }^{126}$ E. Clement, ${ }^{126}$ D. Cussans, ${ }^{126}$ O. Davignon, ${ }^{126}$ H. Flacher, ${ }^{126}$ J. Goldstein, ${ }^{126}$ G. P. Heath, ${ }^{126}$ H. F. Heath, ${ }^{126}$ L. Kreczko, ${ }^{126}$ D. M. Newbold, ${ }^{126, k k k}$ S. Paramesvaran, ${ }^{126}$ T. Sakuma, ${ }^{126}$ S. Seif El Nasr-storey, ${ }^{126}$ D. Smith, ${ }^{126}$ V. J. Smith, ${ }^{126}$ K. W. Bell, ${ }^{127}$ A. Belyaev, ${ }^{127,111}$ C. Brew, ${ }^{127}$ R. M. Brown, ${ }^{127}$ L. Calligaris, ${ }^{127}$ D. Cieri, ${ }^{127}$ D. J. A. Cockerill,,${ }^{127}$ J. A. Coughlan, ${ }^{127}$ K. Harder, ${ }^{127}$ S. Harper, ${ }^{127}$ E. Olaiya, ${ }^{127}$ D. Petyt, ${ }^{127}$ C. H. Shepherd-Themistocleous, ${ }^{127}$ A. Thea, ${ }^{127}$ I. R. Tomalin, ${ }^{127}$ T. Williams, ${ }^{127}$ G. Auzinger, ${ }^{128}$ R. Bainbridge, ${ }^{128}$ J. Borg, ${ }^{128}$ S. Breeze, ${ }^{128}$ O. Buchmuller, ${ }^{128}$ A. Bundock, ${ }^{128}$ S. Casasso, ${ }^{128}$ M. Citron, ${ }^{128}$ D. Colling, ${ }^{128}$ L. Corpe, ${ }^{128}$ P. Dauncey, ${ }^{128}$ G. Davies, ${ }^{128}$ A. De Wit, ${ }^{128}$ M. Della Negra, ${ }^{128}$ R. Di Maria, ${ }^{128}$ A. Elwood, ${ }^{128}$ Y. Haddad, ${ }^{128}$ G. Hall, ${ }^{128}$ G. Iles, ${ }^{128}$ T. James, ${ }^{128}$ R. Lane, ${ }^{128}$ C. Laner, ${ }^{128}$ L. Lyons, ${ }^{128}$ A.-M. Magnan, ${ }^{128}$ S. Malik, ${ }^{128}$ L. Mastrolorenzo, ${ }^{128}$ T. Matsushita, ${ }^{128}$ J. Nash, ${ }^{128}$ A. Nikitenko, ${ }^{128, \mathrm{~h}}$ V. Palladino, ${ }^{128} \mathrm{M}$. Pesaresi, ${ }^{128}$ D. M. Raymond, ${ }^{128}$ A. Richards, ${ }^{128}$ A. Rose, ${ }^{128}$ E. Scott, ${ }^{128}$ C. Seez, ${ }^{128}$ A. Shtipliyski, ${ }^{128}$ S. Summers, ${ }^{128}$ A. Tapper, ${ }^{128}$ K. Uchida, ${ }^{128}$ M. Vazquez Acosta, ${ }^{128, m m m}$ T. Virdee, ${ }^{128, \mathrm{r}}$ N. Wardle, ${ }^{128}$ D. Winterbottom, ${ }^{128}$ J. Wright,${ }^{128}$ S. C. Zenz, ${ }^{128}$ J. E. Cole, ${ }^{129}$ P. R. Hobson, ${ }^{129}$ A. Khan, ${ }^{129}$ P. Kyberd, ${ }^{129}$ I. D. Reid, ${ }^{129}$ P. Symonds, ${ }^{129}$ L. Teodorescu, ${ }^{129}$ M. Turner, ${ }^{129}$ S. Zahid, ${ }^{129}$ A. Borzou, ${ }^{130}$ K. Call, ${ }^{130}$ J. Dittmann, ${ }^{130}$ K. Hatakeyama, ${ }^{130}$ H. Liu, ${ }^{130}$ N. Pastika, ${ }^{130}$ C. Smith, ${ }^{130}$ R. Bartek, ${ }^{131}$ A. Dominguez, ${ }^{131}$ A. Buccilli, ${ }^{132}$ S. I. Cooper, ${ }^{132}$ C. Henderson, ${ }^{132}$ P. Rumerio, ${ }^{132}$ C. West, ${ }^{132}$ D. Arcaro, ${ }^{133}$ A. Avetisyan, ${ }^{133}$ T. Bose, ${ }^{133}$ D. Gastler, ${ }^{133}$ D. Rankin, ${ }^{133}$ C. Richardson, ${ }^{133}$ J. Rohlf, ${ }^{133}$ L. Sulak, ${ }^{133}$ D. Zou, ${ }^{133}$ G. Benelli, ${ }^{134}$ D. Cutts, ${ }^{134}$ A. Garabedian, ${ }^{134}$ M. Hadley, ${ }^{134}$ J. Hakala, ${ }^{134}$ U. Heintz, ${ }^{134}$ J. M. Hogan, ${ }^{134}$ K. H. M. Kwok, ${ }^{134}$ E. Laird, ${ }^{134}$ G. Landsberg, ${ }^{134}$ J. Lee, ${ }^{134}$ Z. Mao, ${ }^{134}$ M. Narain, ${ }^{134}$ J. Pazzini, ${ }^{134}$ S. Piperov, ${ }^{134}$ S. Sagir,${ }^{134}$ R. Syarif, ${ }^{134}$ D. Yu,${ }^{134}$ R. Band,${ }^{135}$ C. Brainerd, ${ }^{135}$ D. Burns, ${ }^{135}$ M. Calderon De La Barca Sanchez, ${ }^{135}$ M. Chertok, ${ }^{135}$ J. Conway, ${ }^{135}$ R. Conway, ${ }^{135}$ P. T. Cox, ${ }^{135}$ R. Erbacher, ${ }^{135}$ C. Flores, ${ }^{135}$ G. Funk, ${ }^{135}$ M. Gardner, ${ }^{135}$ W. Ko, ${ }^{135}$ R. Lander, ${ }^{135}$ C. Mclean, ${ }^{135}$ M. Mulhearn, ${ }^{135}$ D. Pellett, ${ }^{135}$ J. Pilot, ${ }^{135}$ S. Shalhout, ${ }^{135}$ M. Shi, ${ }^{135}$ J. Smith, ${ }^{135}$ D. Stolp, ${ }^{135}$ K. Tos, ${ }^{135}$ M. Tripathi, ${ }^{135}$ Z. Wang, ${ }^{135}$ M. Bachtis, ${ }^{136}$ C. Bravo, ${ }^{136}$ R. Cousins, ${ }^{136}$ A. Dasgupta, ${ }^{136}$ A. Florent, ${ }^{136}$ J. Hauser, ${ }^{136}$ M. Ignatenko, ${ }^{136}$ N. Mccoll, ${ }^{136}$ S. Regnard, ${ }^{136}$ D. Saltzberg, ${ }^{136}$ C. Schnaible, ${ }^{136}$ V. Valuev, ${ }^{136}$ E. Bouvier,${ }^{137}$ K. Burt, ${ }^{137}$ R. Clare, ${ }^{137}$ J. Ellison, ${ }^{137}$ J. W. Gary, ${ }^{137}$ S. M. A. Ghiasi Shirazi, ${ }^{137}$ G. Hanson, ${ }^{137}$ J. Heilman, ${ }^{137}$ E. Kennedy, ${ }^{137}$ F. Lacroix, ${ }^{137}$ O. R. Long ${ }^{137}$ M. Olmedo Negrete, ${ }^{137}$ M. I. Paneva, ${ }^{137}$ W. Si, ${ }^{137}$ L. Wang, ${ }^{137}$ H. Wei, ${ }^{137}$ S. Wimpenny, ${ }^{137}$ B. R. Yates, ${ }^{137}$ J. G. Branson, ${ }^{138}$ S. Cittolin, ${ }^{138}$ M. Derdzinski, ${ }^{138}$ R. Gerosa, ${ }^{138}$ D. Gilbert, ${ }^{138}$ B. Hashemi, ${ }^{138}$ A. Holzner, ${ }^{138}$ D. Klein, ${ }^{138}$ G. Kole, ${ }^{138}$ V. Krutelyov, ${ }^{138}$ J. Letts, ${ }^{138}$ I. Macneill, ${ }^{138}$ M. Masciovecchio, ${ }^{138}$ D. Olivito, ${ }^{138}$ S. Padhi, ${ }^{138}$ M. Pieri, ${ }^{138}$ M. Sani, ${ }^{138}$ V. Sharma, ${ }^{138}$ S. Simon, ${ }^{138}$ M. Tadel, ${ }^{138}$ A. Vartak, ${ }^{138}$ S. Wasserbaech, ${ }^{138, n n n}$ J. Wood, ${ }^{138}$ F. Würthwein, ${ }^{138}$ A. Yagil, ${ }^{138}$ G. Zevi Della Porta, ${ }^{138}$ N. Amin, ${ }^{139}$ R. Bhandari, ${ }^{139}$ J. Bradmiller-Feld, ${ }^{139}$ C. Campagnari, ${ }^{139}$ A. Dishaw, ${ }^{139}$ V. Dutta, ${ }^{139}$ M. Franco Sevilla, ${ }^{139}$ C. George, ${ }^{139}$ 
F. Golf, ${ }^{139}$ L. Gouskos, ${ }^{139}$ J. Gran, ${ }^{139}$ R. Heller, ${ }^{139}$ J. Incandela, ${ }^{139}$ S. D. Mullin, ${ }^{139}$ A. Ovcharova, ${ }^{139}$ H. Qu, ${ }^{139}$ J. Richman, ${ }^{139}$ D. Stuart, ${ }^{139}$ I. Suarez, ${ }^{139}$ J. Yoo, ${ }^{139}$ D. Anderson, ${ }^{140}$ A. Bornheim, ${ }^{140}$ J. M. Lawhorn, ${ }^{140}$ H. B. Newman, ${ }^{140}$ T. Nguyen, ${ }^{140}$ C. Pena, ${ }^{140}$ M. Spiropulu, ${ }^{140}$ J. R. Vlimant, ${ }^{140}$ S. Xie, ${ }^{140}$ Z. Zhang, ${ }^{140}$ R. Y. Zhu, ${ }^{140}$ M. B. Andrews, ${ }^{141}$

T. Ferguson, ${ }^{141}$ T. Mudholkar, ${ }^{141}$ M. Paulini, ${ }^{141}$ J. Russ, ${ }^{141}$ M. Sun,${ }^{141}$ H. Vogel,${ }^{141}$ I. Vorobiev, ${ }^{141}$ M. Weinberg, ${ }^{141}$ J. P. Cumalat, ${ }^{142}$ W. T. Ford, ${ }^{142}$ F. Jensen, ${ }^{142}$ A. Johnson, ${ }^{142}$ M. Krohn, ${ }^{142}$ S. Leontsinis, ${ }^{142}$ T. Mulholland,${ }^{142}$ K. Stenson, ${ }^{142}$ S. R. Wagner, ${ }^{142}$ J. Alexander, ${ }^{143}$ J. Chaves,${ }^{143}$ J. Chu, ${ }^{143}$ S. Dittmer, ${ }^{143}$ K. Mcdermott, ${ }^{143}$ N. Mirman, ${ }^{143}$ J. R. Patterson, ${ }^{143}$ D. Quach, ${ }^{143}$ A. Rinkevicius, ${ }^{143}$ A. Ryd, ${ }^{143}$ L. Skinnari, ${ }^{143}$ L. Soffi, ${ }^{143}$ S. M. Tan, ${ }^{143}$ Z. Tao, ${ }^{143}$ J. Thom, ${ }^{143}$ J. Tucker, ${ }^{143}$ P. Wittich, ${ }^{143}$ M. Zientek, ${ }^{143}$ S. Abdullin, ${ }^{144}$ M. Albrow, ${ }^{144}$ M. Alyari, ${ }^{144}$ G. Apollinari, ${ }^{144}$ A. Apresyan, ${ }^{144}$ A. Apyan, ${ }^{144}$

S. Banerjee, ${ }^{144}$ L. A. T. Bauerdick, ${ }^{144}$ A. Beretvas, ${ }^{144}$ J. Berryhill, ${ }^{144}$ P. C. Bhat ${ }^{144}$ G. Bolla, ${ }^{144, a}$ K. Burkett, ${ }^{144}$

J. N. Butler, ${ }^{144}$ A. Canepa, ${ }^{144}$ G. B. Cerati, ${ }^{144}$ H. W. K. Cheung, ${ }^{144}$ F. Chlebana, ${ }^{144}$ M. Cremonesi, ${ }^{144}$ J. Duarte, ${ }^{144}$ V. D. Elvira ${ }^{144}$ J. Freeman, ${ }^{144}$ Z. Gecse ${ }^{144}$ E. Gottschalk, ${ }^{144}$ L. Gray, ${ }^{144}$ D. Green,${ }^{144}$ S. Grünendahl,${ }^{144}$ O. Gutsche, ${ }^{144}$ R. M. Harris, ${ }^{144}$ S. Hasegawa, ${ }^{144}$ J. Hirschauer, ${ }^{144}$ Z. Hu, ${ }^{144}$ B. Jayatilaka, ${ }^{144}$ S. Jindariani, ${ }^{144}$ M. Johnson, ${ }^{144}$ U. Joshi, ${ }^{144}$ B. Klima, ${ }^{144}$ B. Kreis, ${ }^{144}$ S. Lammel, ${ }^{144}$ D. Lincoln,${ }^{144}$ R. Lipton, ${ }^{144}$ M. Liu, ${ }^{144}$ T. Liu, ${ }^{144}$ R. Lopes De Sá,${ }^{144}$ J. Lykken, ${ }^{144}$ K. Maeshima, ${ }^{144}$ N. Magini, ${ }^{144}$ J. M. Marraffino, ${ }^{144}$ D. Mason, ${ }^{144}$ P. McBride,${ }^{144}$ P. Merkel, ${ }^{144}$ S. Mrenna, ${ }^{144}$ S. Nahn, ${ }^{144}$ V. O’Dell,,${ }^{144}$ K. Pedro, ${ }^{144}$ O. Prokofyev, ${ }^{144}$ G. Rakness, ${ }^{144}$ L. Ristori, ${ }^{144}$ B. Schneider, ${ }^{144}$ E. Sexton-Kennedy, ${ }^{144}$ A. Soha, ${ }^{144}$ W. J. Spalding, ${ }^{144}$ L. Spiegel, ${ }^{144}$ S. Stoynev, ${ }^{144}$ J. Strait, ${ }^{144}$ N. Strobbe,${ }^{144}$ L. Taylor,${ }^{144}$ S. Tkaczyk, ${ }^{144}$ N. V. Tran,${ }^{144}$

L. Uplegger, ${ }^{144}$ E. W. Vaandering, ${ }^{144}$ C. Vernieri, ${ }^{144}$ M. Verzocchi, ${ }^{144}$ R. Vidal, ${ }^{144}$ M. Wang, ${ }^{144}$ H. A. Weber, ${ }^{144}$ A. Whitbeck, ${ }^{144}$ D. Acosta, ${ }^{145}$ P. Avery, ${ }^{145}$ P. Bortignon, ${ }^{145}$ D. Bourilkov, ${ }^{145}$ A. Brinkerhoff, ${ }^{145}$ A. Carnes, ${ }^{145}$ M. Carver, ${ }^{145}$ D. Curry, ${ }^{145}$ R. D. Field, ${ }^{145}$ I. K. Furic, ${ }^{145}$ S. V. Gleyzer, ${ }^{145}$ B. M. Joshi, ${ }^{145}$ J. Konigsberg, ${ }^{145}$ A. Korytov, ${ }^{145}$ K. Kotov, ${ }^{145}$ P. Ma, ${ }^{145}$ K. Matchev, ${ }^{145}$ H. Mei, ${ }^{145}$ G. Mitselmakher, ${ }^{145}$ D. Rank, ${ }^{145}$ K. Shi,${ }^{145}$ D. Sperka, ${ }^{145}$ N. Terentyev,${ }^{145}$ L. Thomas, ${ }^{145}$ J. Wang, ${ }^{145}$ S. Wang, ${ }^{145}$ J. Yelton, ${ }^{145}$ Y. R. Joshi, ${ }^{146}$ S. Linn, ${ }^{146}$ P. Markowitz, ${ }^{146}$ J. L. Rodriguez, ${ }^{146}$ A. Ackert, ${ }^{147}$ T. Adams, ${ }^{147}$ A. Askew, ${ }^{147}$ S. Hagopian, ${ }^{147}$ V. Hagopian, ${ }^{147}$ K. F. Johnson, ${ }^{147}$ T. Kolberg, ${ }^{147}$ G. Martinez, ${ }^{147}$ T. Perry, ${ }^{147}$ H. Prosper, ${ }^{147}$ A. Saha, ${ }^{147}$ A. Santra, ${ }^{147}$ V. Sharma, ${ }^{147}$ R. Yohay, ${ }^{147}$ M. M. Baarmand, ${ }^{148}$ V. Bhopatkar, ${ }^{148}$

S. Colafranceschi, ${ }^{148}$ M. Hohlmann, ${ }^{148}$ D. Noonan, ${ }^{148}$ T. Roy, ${ }^{148}$ F. Yumiceva, ${ }^{148}$ M. R. Adams, ${ }^{149}$ L. Apanasevich, ${ }^{149}$ D. Berry, ${ }^{149}$ R. R. Betts, ${ }^{149}$ R. Cavanaugh, ${ }^{149}$ X. Chen, ${ }^{149}$ O. Evdokimov, ${ }^{149}$ C. E. Gerber, ${ }^{149}$ D. A. Hangal, ${ }^{149}$ D. J. Hofman, ${ }^{149}$ K. Jung, ${ }^{149}$ J. Kamin, ${ }^{149}$ I. D. Sandoval Gonzalez, ${ }^{149}$ M. B. Tonjes, ${ }^{149}$ H. Trauger, ${ }^{149}$ N. Varelas, ${ }^{149}$ H. Wang, ${ }^{149}$ Z. Wu ${ }^{149}$ J. Zhang, ${ }^{149}$ B. Bilki, ${ }^{150,000}$ W. Clarida, ${ }^{150}$ K. Dilsiz, ${ }^{150, p p p}$ S. Durgut, ${ }^{150}$ R. P. Gandrajula, ${ }^{150}$ M. Haytmyradov, ${ }^{150}$ V. Khristenko, ${ }^{150}$ J.-P. Merlo ${ }^{150}$ H. Mermerkaya, ${ }^{150, q q 9}$ A. Mestvirishvili, ${ }^{150}$ A. Moeller, ${ }^{150}$ J. Nachtman, ${ }^{150}$ H. Ogul, ${ }^{150, r r}$ Y. Onel, ${ }^{150}$ F. Ozok, ${ }^{150, \text { sss }}$ A. Penzo, ${ }^{150}$ C. Snyder, ${ }^{150}$ E. Tiras, ${ }^{150}$ J. Wetzel ${ }^{150}$ K. Yi, ${ }^{150}$ B. Blumenfeld, ${ }^{151}$ A. Cocoros, ${ }^{151}$ N. Eminizer ${ }^{151}$ D. Fehling, ${ }^{151}$ L. Feng, ${ }^{151}$ A. V. Gritsan, ${ }^{151}$ P. Maksimovic, ${ }^{151}$ J. Roskes, ${ }^{151}$ U. Sarica ${ }^{151}$ M. Swartz ${ }^{151}$ M. Xiao, ${ }^{151}$ C. You, ${ }^{151}$ A. Al-bataineh, ${ }^{152}$ P. Baringer, ${ }^{152}$ A. Bean, ${ }^{152}$ S. Boren, ${ }^{152}$ J. Bowen, ${ }^{152}$ J. Castle, ${ }^{152}$ S. Khalil, ${ }^{152}$ A. Kropivnitskaya, ${ }^{152}$ D. Majumder, ${ }^{152}$ W. Mcbrayer, ${ }^{152}$ M. Murray, ${ }^{152}$ C. Royon, ${ }^{152}$ S. Sanders, ${ }^{152}$

E. Schmitz, ${ }^{152}$ J. D. Tapia Takaki, ${ }^{152}$ Q. Wang, ${ }^{152}$ A. Ivanov, ${ }^{153}$ K. Kaadze, ${ }^{153}$ Y. Maravin, ${ }^{153}$ A. Mohammadi, ${ }^{153}$ L. K. Saini, ${ }^{153}$ N. Skhirtladze, ${ }^{153}$ S. Toda ${ }^{153}$ F. Rebassoo, ${ }^{154}$ D. Wright,${ }^{154}$ C. Anelli, ${ }^{155}$ A. Baden ${ }^{155}$ O. Baron, ${ }^{155}$ A. Belloni, ${ }^{155}$ B. Calvert, ${ }^{155}$ S. C. Eno, ${ }^{155}$ Y. Feng, ${ }^{155}$ C. Ferraioli, ${ }^{155}$ N. J. Hadley, ${ }^{155}$ S. Jabeen, ${ }^{155}$ G. Y. Jeng, ${ }^{155}$ R. G. Kellogg, ${ }^{155}$ J. Kunkle, ${ }^{155}$ A. C. Mignerey, ${ }^{155}$ F. Ricci-Tam, ${ }^{155}$ Y. H. Shin, ${ }^{155}$ A. Skuja, ${ }^{155}$ S. C. Tonwar, ${ }^{155}$ D. Abercrombie, ${ }^{156}$ B. Allen, ${ }^{156}$ V. Azzolini, ${ }^{156}$ R. Barbieri, ${ }^{156}$ A. Baty, ${ }^{156}$ R. Bi, ${ }^{156}$ S. Brandt, ${ }^{156}$ W. Busza, ${ }^{156}$ I. A. Cali, ${ }^{156}$ M. D’Alfonso, ${ }^{156}$ Z. Demiragli, ${ }^{156}$ G. Gomez Ceballos, ${ }^{156}$ M. Goncharov, ${ }^{156}$ D. Hsu, ${ }^{156} \mathrm{M}$. Hu, ${ }^{156}$ Y. Iiyama, ${ }^{156}$ G. M. Innocenti, ${ }^{156}$ M. Klute, ${ }^{156}$ D. Kovalskyi, ${ }^{156}$ Y. S. Lai, ${ }^{156}$ Y.-J. Lee, ${ }^{156}$ A. Levin, ${ }^{156}$ P. D. Luckey, ${ }^{156}$ B. Maier,${ }^{156}$ A. C. Marini, ${ }^{156}$ C. Mcginn, ${ }^{156}$ C. Mironov, ${ }^{156}$ S. Narayanan, ${ }^{156}$ X. Niu, ${ }^{156}$ C. Paus, ${ }^{156}$ C. Roland,${ }^{156}$ G. Roland, ${ }^{156}$ J. Salfeld-Nebgen, ${ }^{156}$ G. S. F. Stephans, ${ }^{156}$ K. Tatar, ${ }^{156}$ D. Velicanu, ${ }^{156}$ J. Wang, ${ }^{156}$ T. W. Wang, ${ }^{156}$ B. Wyslouch, ${ }^{156}$ A. C. Benvenuti, ${ }^{157}$ R. M. Chatterjee, ${ }^{157}$ A. Evans, ${ }^{157}$ P. Hansen, ${ }^{157}$ J. Hiltbrand, ${ }^{157}$ S. Kalafut, ${ }^{157}$ Y. Kubota, ${ }^{157}$ Z. Lesko, ${ }^{157}$

J. Mans, ${ }^{157}$ S. Nourbakhsh, ${ }^{157}$ N. Ruckstuhl,,${ }^{157}$ R. Rusack, ${ }^{157}$ J. Turkewitz,${ }^{157}$ M. A. Wadud ${ }^{157}$ J. G. Acosta, ${ }^{158}$

S. Oliveros, ${ }^{158}$ E. Avdeeva, ${ }^{159}$ K. Bloom, ${ }^{159}$ D. R. Claes, ${ }^{159}$ C. Fangmeier, ${ }^{159}$ R. Gonzalez Suarez, ${ }^{159}$ R. Kamalieddin, ${ }^{159}$ I. Kravchenko, ${ }^{159}$ J. Monroy, ${ }^{159}$ J. E. Siado, ${ }^{159}$ G. R. Snow, ${ }^{159}$ B. Stieger, ${ }^{159}$ J. Dolen, ${ }^{160}$ A. Godshalk, ${ }^{160}$ C. Harrington, ${ }^{160}$ I. Iashvili, ${ }^{160}$ D. Nguyen, ${ }^{160}$ A. Parker, ${ }^{160}$ S. Rappoccio, ${ }^{160}$ B. Roozbahani, ${ }^{160}$ G. Alverson, ${ }^{161}$ E. Barberis, ${ }^{161}$

A. Hortiangtham, ${ }^{161}$ A. Massironi, ${ }^{161}$ D. M. Morse, ${ }^{161}$ T. Orimoto, ${ }^{161}$ R. Teixeira De Lima,${ }^{161}$ D. Trocino, ${ }^{161}$ D. Wood, ${ }^{161}$ S. Bhattacharya, ${ }^{162}$ O. Charaf, ${ }^{162}$ K. A. Hahn, ${ }^{162}$ N. Mucia, ${ }^{162}$ N. Odell, ${ }^{162}$ B. Pollack, ${ }^{162}$ M. H. Schmitt, ${ }^{162}$ K. Sung, ${ }^{162}$ M. Trovato, ${ }^{162}$ M. Velasco, ${ }^{162}$ N. Dev, ${ }^{163}$ M. Hildreth, ${ }^{163}$ K. Hurtado Anampa, ${ }^{163}$ C. Jessop, ${ }^{163}$ D. J. Karmgard, ${ }^{163}$ 
N. Kellams, ${ }^{163}$ K. Lannon, ${ }^{163}$ N. Loukas, ${ }^{163}$ N. Marinelli, ${ }^{163}$ F. Meng, ${ }^{163}$ C. Mueller, ${ }^{163}$ Y. Musienko, ${ }^{163, m m}$ M. Planer, ${ }^{163}$ A. Reinsvold, ${ }^{163}$ R. Ruchti, ${ }^{163}$ G. Smith, ${ }^{163}$ S. Taroni, ${ }^{163}$ M. Wayne, ${ }^{163}$ M. Wolf, ${ }^{163}$ A. Woodard ${ }^{163}$ J. Alimena, ${ }^{164}$ L. Antonelli, ${ }^{164}$ B. Bylsma, ${ }^{164}$ L. S. Durkin, ${ }^{164}$ S. Flowers, ${ }^{164}$ B. Francis, ${ }^{164}$ A. Hart, ${ }^{164}$ C. Hill, ${ }^{164}$ W. Ji, ${ }^{164}$ B. Liu, ${ }^{164}$ W. Luo, ${ }^{164}$ D. Puigh, ${ }^{164}$ B. L. Winer, ${ }^{164}$ H. W. Wulsin, ${ }^{164}$ S. Cooperstein, ${ }^{165}$ O. Driga, ${ }^{165}$ P. Elmer, ${ }^{165}$ J. Hardenbrook, ${ }^{165}$ P. Hebda, ${ }^{165}$ S. Higginbotham, ${ }^{165}$ D. Lange, ${ }^{165}$ J. Luo, ${ }^{165}$ D. Marlow, ${ }^{165}$ K. Mei, ${ }^{165}$ I. Ojalvo, ${ }^{165}$ J. Olsen, ${ }^{165}$ C. Palmer, ${ }^{165}$ P. Piroué, ${ }^{165}$ D. Stickland, ${ }^{165}$ C. Tully, ${ }^{165}$ S. Malik, ${ }^{166}$ S. Norberg, ${ }^{166}$ A. Barker,${ }^{167}$ V. E. Barnes, ${ }^{167}$ S. Das,${ }^{167}$ S. Folgueras, ${ }^{167}$ L. Gutay, ${ }^{167}$ M. K. Jha ${ }^{167}$ M. Jones ${ }^{167}$ A. W. Jung, ${ }^{167}$ A. Khatiwada ${ }^{167}$ D. H. Miller, ${ }^{167}$ N. Neumeister,${ }^{167}$ C. C. Peng, ${ }^{167}$ H. Qiu, ${ }^{167}$ J. F. Schulte, ${ }^{167}$ J. Sun, ${ }^{167}$ F. Wang, ${ }^{167}$ W. Xie, ${ }^{167}$ T. Cheng, ${ }^{168}$ N. Parashar, ${ }^{168}$ J. Stupak, ${ }^{168}$ A. Adair, ${ }^{169}$ Z. Chen, ${ }^{169}$ K. M. Ecklund, ${ }^{169}$ S. Freed, ${ }^{169}$ F. J. M. Geurts, ${ }^{169}$ M. Guilbaud, ${ }^{169}$ M. Kilpatrick, ${ }^{169}$ W. Li, ${ }^{169}$ B. Michlin, ${ }^{169}$ M. Northup, ${ }^{169}$ B. P. Padley, ${ }^{169}$ J. Roberts, ${ }^{169}$ J. Rorie, ${ }^{169}$ W. Shi, ${ }^{169}$ Z. Tu, ${ }^{169}$ J. Zabel, ${ }^{169}$ A. Zhang, ${ }^{169}$ A. Bodek, ${ }^{170}$ P. de Barbaro, ${ }^{170}$ R. Demina, ${ }^{170}$ Y. t. Duh, ${ }^{170}$ T. Ferbel,${ }^{170}$ M. Galanti, ${ }^{170}$ A. Garcia-Bellido, ${ }^{170}$ J. Han, ${ }^{170}$ O. Hindrichs,${ }^{170}$

A. Khukhunaishvili, ${ }^{170}$ K. H. Lo,${ }^{170}$ P. Tan, ${ }^{170}$ M. Verzetti, ${ }^{170}$ R. Ciesielski, ${ }^{171}$ K. Goulianos, ${ }^{171}$ C. Mesropian, ${ }^{171}$ A. Agapitos ${ }^{172}$ J. P. Chou, ${ }^{172}$ Y. Gershtein, ${ }^{172}$ T. A. Gómez Espinosa, ${ }^{172}$ E. Halkiadakis, ${ }^{172}$ M. Heindl,${ }^{172}$ E. Hughes,${ }^{172}$ S. Kaplan, ${ }^{172}$ R. Kunnawalkam Elayavalli, ${ }^{172}$ S. Kyriacou, ${ }^{172}$ A. Lath,${ }^{172}$ R. Montalvo, ${ }^{172}$ K. Nash, ${ }^{172}$ M. Osherson, ${ }^{172}$ H. Saka, ${ }^{172}$ S. Salur, ${ }^{172}$ S. Schnetzer, ${ }^{172}$ D. Sheffield, ${ }^{172}$ S. Somalwar, ${ }^{172}$ R. Stone, ${ }^{172}$ S. Thomas, ${ }^{172}$ P. Thomassen, ${ }^{172}$ M. Walker, ${ }^{172}$ A. G. Delannoy, ${ }^{173}$ M. Foerster, ${ }^{173}$ J. Heideman, ${ }^{173}$ G. Riley, ${ }^{173}$ K. Rose, ${ }^{173}$ S. Spanier, ${ }^{173}$ K. Thapa, ${ }^{173}$ O. Bouhali, ${ }^{174, \text { ttt }}$ A. Castaneda Hernandez, ${ }^{174, \text { ttt }}$ A. Celik, ${ }^{174}$ M. Dalchenko, ${ }^{174}$ M. De Mattia, ${ }^{174}$ A. Delgado, ${ }^{174}$ S. Dildick, ${ }^{174}$ R. Eusebi, ${ }^{174}$ J. Gilmore ${ }^{174}$ T. Huang, ${ }^{174}$ T. Kamon, ${ }^{174, \text { uuu }}$ R. Mueller, ${ }^{174}$ Y. Pakhotin, ${ }^{174}$ R. Patel, ${ }^{174}$ A. Perloff, ${ }^{174}$ L. Perniè, ${ }^{174}$ D. Rathjens, ${ }^{174}$ A. Safonov,${ }^{174}$ A. Tatarinov, ${ }^{174}$ K. A. Ulmer, ${ }^{174}$ N. Akchurin, ${ }^{175}$ J. Damgov, ${ }^{175}$ F. De Guio, ${ }^{175}$ P. R. Dudero, ${ }^{175}$ J. Faulkner, ${ }^{175}$ E. Gurpinar, ${ }^{175}$ S. Kunori, ${ }^{175}$ K. Lamichhane,${ }^{175}$ S. W. Lee, ${ }^{175}$ T. Libeiro, ${ }^{175}$ T. Mengke, ${ }^{175}$ S. Muthumuni, ${ }^{175}$ T. Peltola, ${ }^{175}$ S. Undleeb, ${ }^{175}$ I. Volobouev, ${ }^{175}$ Z. Wang, ${ }^{175}$ S. Greene, ${ }^{176}$ A. Gurrola, ${ }^{176}$ R. Janjam, ${ }^{176}$ W. Johns, ${ }^{176}$ C. Maguire, ${ }^{176}$ A. Melo, ${ }^{176} \mathrm{H}$. Ni, ${ }^{176}$ K. Padeken, ${ }^{176}$ P. Sheldon, ${ }^{176}$ S. Tuo, ${ }^{176}$ J. Velkovska, ${ }^{176}$ Q. Xu, ${ }^{176}$ M. W. Arenton, ${ }^{177}$ P. Barria, ${ }^{177}$ B. Cox,${ }^{177}$ R. Hirosky, ${ }^{177}$ M. Joyce, ${ }^{177}$ A. Ledovskoy, ${ }^{177}$ H. Li, ${ }^{177}$ C. Neu, ${ }^{177}$ T. Sinthuprasith, ${ }^{177}$ Y. Wang, ${ }^{177}$ E. Wolfe,${ }^{177}$ F. Xia, ${ }^{177}$ R. Harr, ${ }^{178}$ P. E. Karchin, ${ }^{178}$ N. Poudyal, ${ }^{178}$ J. Sturdy,${ }^{178}$ P. Thapa, ${ }^{178}$ S. Zaleski, ${ }^{178}$ M. Brodski, ${ }^{179}$ J. Buchanan, ${ }^{179}$ C. Caillol, ${ }^{179}$ S. Dasu, ${ }^{179}$ L. Dodd,${ }^{179}$ S. Duric,${ }^{179}$ B. Gomber, ${ }^{179}$ M. Grothe, ${ }^{179}$ M. Herndon, ${ }^{179}$ A. Hervé, ${ }^{179}$ U. Hussain, ${ }^{179}$ P. Klabbers, ${ }^{179}$ A. Lanaro, ${ }^{179}$ A. Levine, ${ }^{179}$ K. Long, ${ }^{179}$ R. Loveless, ${ }^{179}$ G. Polese, ${ }^{179}$ T. Ruggles, ${ }^{179}$ A. Savin, ${ }^{179}$ N. Smith, ${ }^{179}$ W. H. Smith, ${ }^{179}$ D. Taylor, ${ }^{179}$ and N. Woods ${ }^{179}$

(CMS Collaboration)

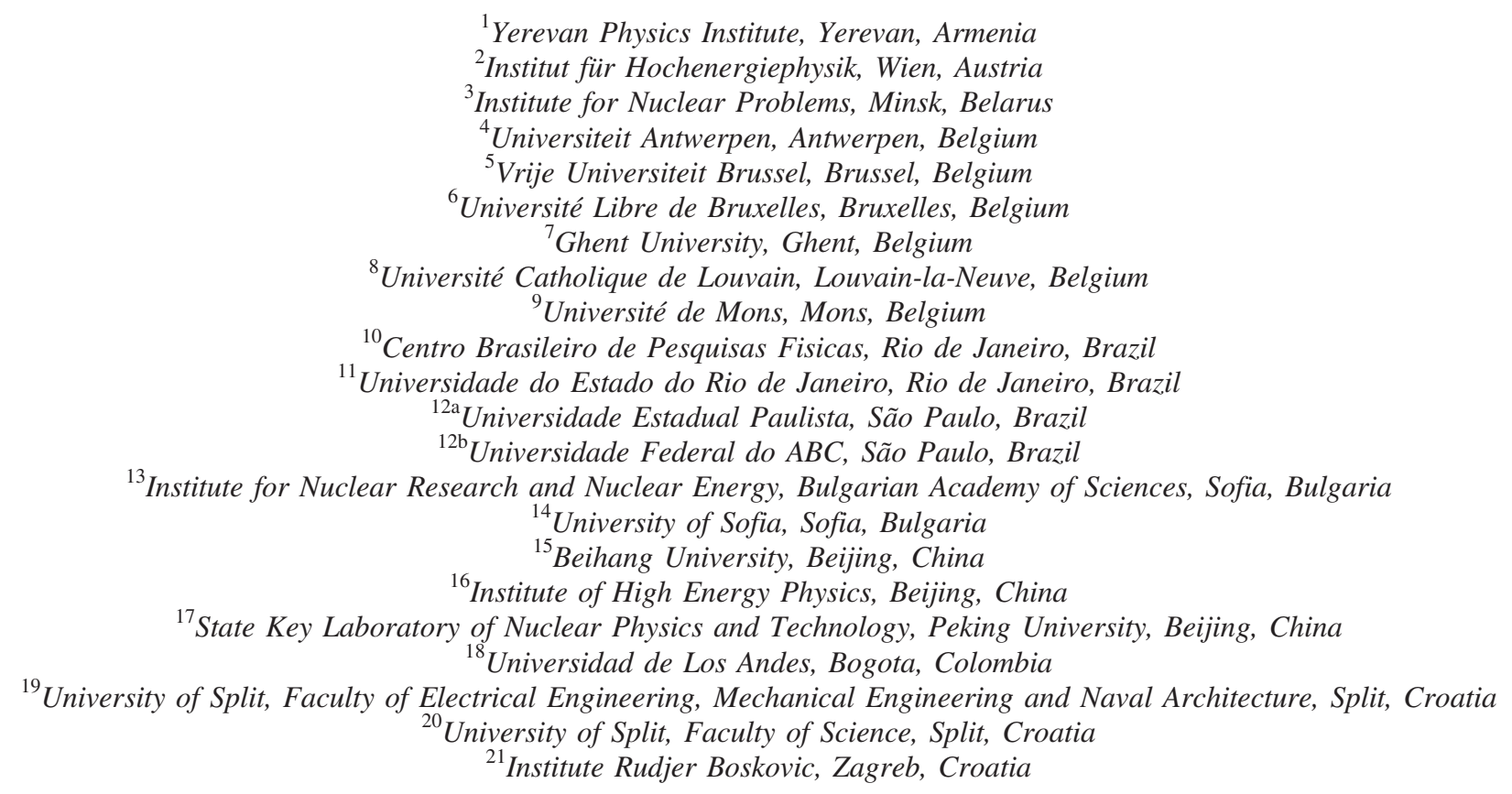




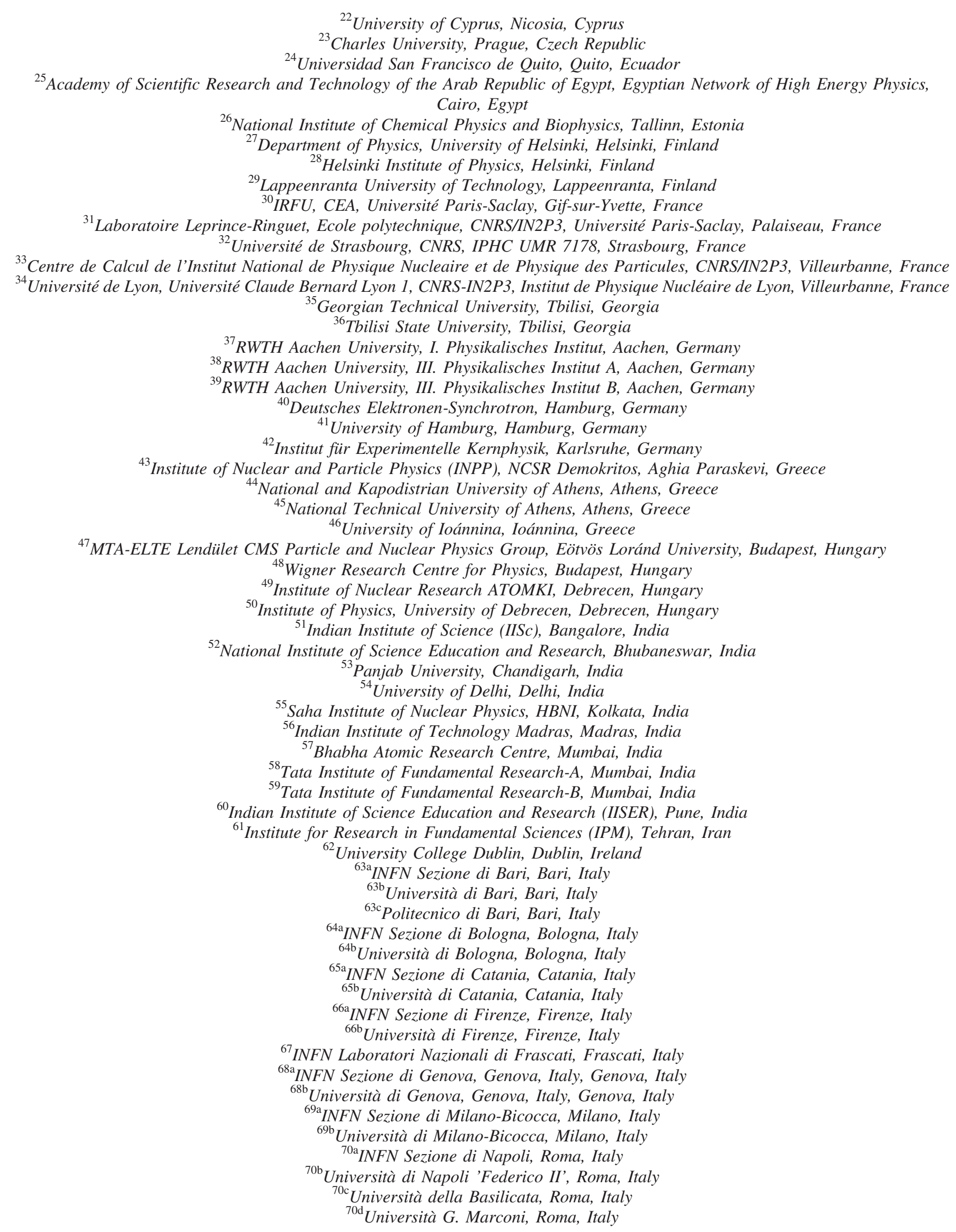




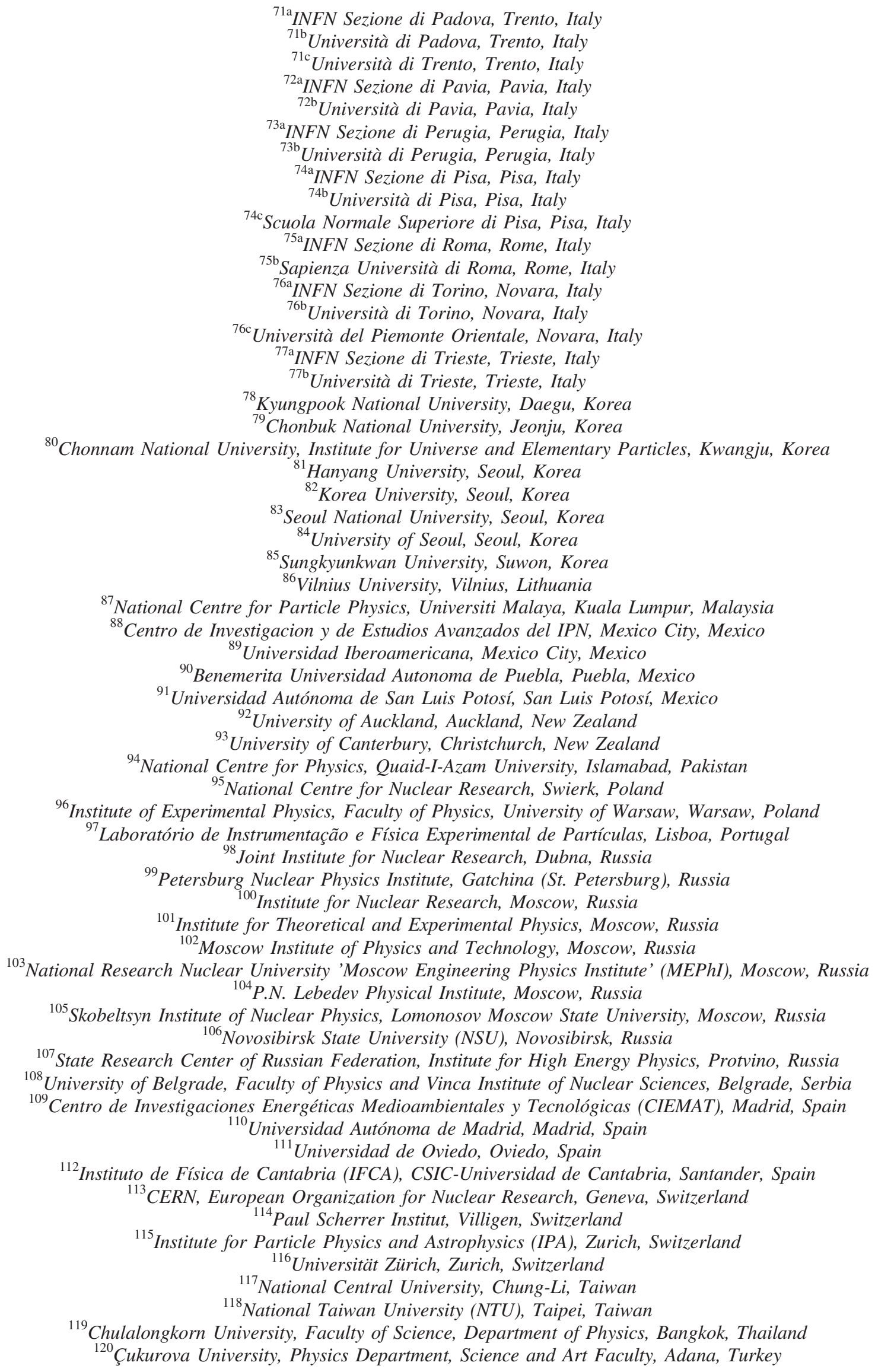


${ }^{121}$ Middle East Technical University, Physics Department, Ankara, Turkey

${ }^{122}$ Bogazici University, Istanbul, Turkey

${ }^{123}$ Istanbul Technical University, Istanbul, Turkey

${ }^{124}$ Institute for Scintillation Materials of National Academy of Science of Ukraine, Kharkov, Ukraine

${ }^{125}$ National Scientific Center, Kharkov Institute of Physics and Technology, Kharkov, Ukraine

${ }^{126}$ University of Bristol, Bristol, United Kingdom

${ }^{127}$ Rutherford Appleton Laboratory, Didcot, United Kingdom

${ }^{128}$ Imperial College, London, United Kingdom

${ }^{129}$ Brunel University, Uxbridge, United Kingdom

${ }^{130}$ Baylor University, Waco, Texas, USA

${ }^{131}$ Catholic University of America, Washington DC, USA

${ }^{132}$ The University of Alabama, Tuscaloosa, Alabama, USA

${ }^{133}$ Boston University, Boston, Massachusetts, USA

${ }^{134}$ Brown University, Providence, Rhode Island, USA

${ }^{135}$ University of California, Davis, Davis, California, USA

${ }^{136}$ University of California, Los Angeles, California, USA

${ }^{137}$ University of California, Riverside, Riverside, California, USA

${ }^{138}$ University of California, San Diego, La Jolla, California, USA

${ }^{139}$ University of California, Santa Barbara-Department of Physics, Santa Barbara, California, USA

${ }^{140}$ California Institute of Technology, Pasadena, California, USA

${ }^{141}$ Carnegie Mellon University, Pittsburgh, Pennsylvania, USA

${ }^{142}$ University of Colorado Boulder, Boulder, Colorado, USA

${ }^{143}$ Cornell University, Ithaca, New York, USA

${ }^{144}$ Fermi National Accelerator Laboratory, Batavia, Illinois, USA

${ }^{145}$ University of Florida, Gainesville, Florida, USA

${ }^{146}$ Florida International University, Miami, Florida, USA

${ }^{147}$ Florida State University, Tallahassee, Florida, USA

${ }^{148}$ Florida Institute of Technology, Melbourne, Florida, USA

${ }^{149}$ University of Illinois at Chicago (UIC), Chicago, Illinois, USA

${ }^{150}$ The University of Iowa, Iowa City, Iowa, USA

${ }^{151}$ Johns Hopkins University, Baltimore, Maryland, USA

${ }^{152}$ The University of Kansas, Lawrence, Kansas, USA

${ }^{153}$ Kansas State University, Manhattan, Kansas, USA

${ }^{154}$ Lawrence Livermore National Laboratory, Livermore, California, USA

${ }^{155}$ University of Maryland, College Park, Maryland, USA

${ }^{156}$ Massachusetts Institute of Technology, Cambridge, Massachusetts, USA

${ }^{157}$ University of Minnesota, Minneapolis, Minnesota, USA

${ }^{158}$ University of Mississippi, Oxford, Mississippi, USA

${ }^{159}$ University of Nebraska-Lincoln, Lincoln, Nebraska, USA

${ }^{160}$ State University of New York at Buffalo, Buffalo, New York, USA

${ }^{161}$ Northeastern University, Boston, Massachusetts, USA

${ }^{162}$ Northwestern University, Evanston, Illinois, USA

${ }^{163}$ University of Notre Dame, Notre Dame, Indiana, USA

${ }^{164}$ The Ohio State University, Columbus, Ohio, USA

${ }^{165}$ Princeton University, Princeton, New Jersey, USA

${ }^{166}$ University of Puerto Rico, Mayaguez, Puerto Rico, USA

${ }^{167}$ Purdue University, West Lafayette, Indiana, USA

${ }^{168}$ Purdue University Northwest, Hammond, Indiana, USA

${ }^{169}$ Rice University, Houston, Texas, USA

${ }^{170}$ University of Rochester, Rochester, New York, USA

${ }^{171}$ The Rockefeller University, New York, New York, USA

${ }^{172}$ Rutgers, The State University of New Jersey, Piscataway, New Jersey, USA

${ }^{173}$ University of Tennessee, Knoxville, Tennessee, USA

${ }^{174}$ Texas A\&M University, College Station, Texas, USA

${ }^{175}$ Texas Tech University, Lubbock, Texas, USA

${ }^{176}$ Vanderbilt University, Nashville, Tennessee, USA

${ }^{177}$ University of Virginia, Charlottesville, Virginia, USA

${ }^{178}$ Wayne State University, Detroit, Michigan, USA

${ }^{179}$ University of Wisconsin-Madison, Madison, Wisconsin, USA 


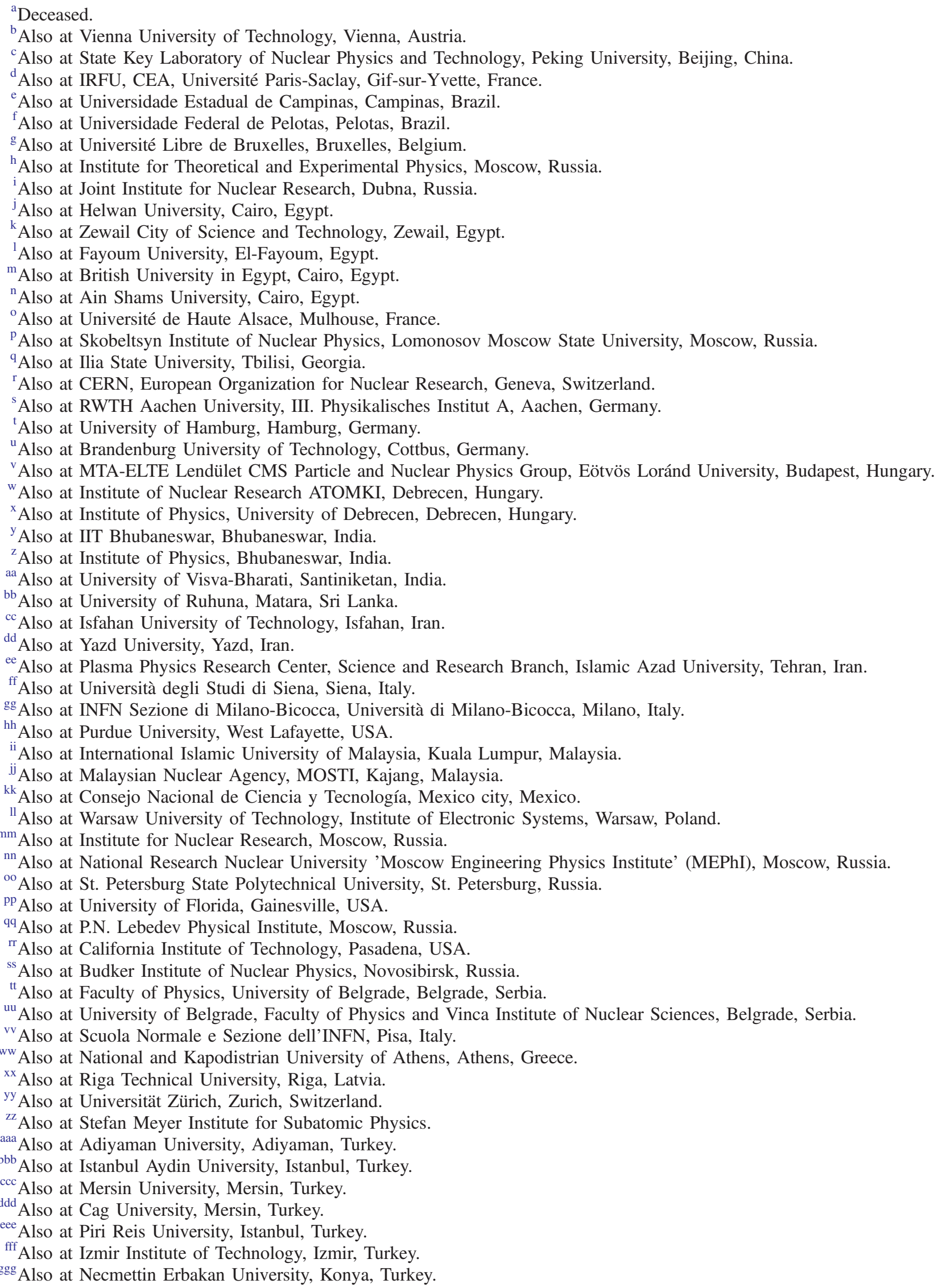




\footnotetext{
${ }^{\text {hhh }}$ Also at Marmara University, Istanbul, Turkey.

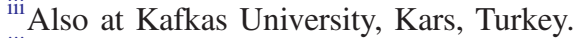

${ }^{\mathrm{jjj}}$ Also at Istanbul Bilgi University, Istanbul, Turkey.

${ }^{k k k}$ Also at Rutherford Appleton Laboratory, Didcot, United Kingdom.

${ }^{111}$ Also at School of Physics and Astronomy, University of Southampton, Southampton, United Kingdom.

${ }^{\mathrm{mmm}}$ Also at Instituto de Astrofísica de Canarias, La Laguna, Spain.

${ }^{n n n}$ Also at Utah Valley University, Orem, USA.

${ }^{\mathrm{ooo}}$ Also at Beykent University.

${ }^{\mathrm{ppp}}$ Also at Bingol University, Bingol, Turkey.

${ }^{\mathrm{qqq}}$ Also at Erzincan University, Erzincan, Turkey.

${ }^{\mathrm{rrr}}$ Also at Sinop University, Sinop, Turkey.

${ }^{\text {sss }}$ Also at Mimar Sinan University, Istanbul, Istanbul, Turkey.

${ }^{t t t}$ Also at Texas A\&M University at Qatar, Doha, Qatar.

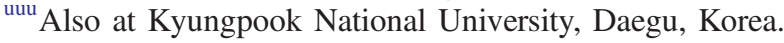

